\title{
A free boundary problem involving a cusp: breakthrough of salt water
}

\author{
H. W. AlT \\ Institut für Angewandte Mathematik, Universität Bonn, Wegelerstrasse 6, 5300 Bonn 1, Germany \\ C. J. VAN DUIJN ${ }^{\dagger}$ \\ Centrum voor Wiskunde en Informatica, Kruislaan 413, 1098 SJ Amsterdam, The Netherlands
}

[Received 23 October 1998]

\begin{abstract}
In this paper we study a two-phase free boundary problem describing the stationary flow of fresh and salt water in a porous medium, when both fluids are drawn into a well. For given discharges at the well ( $Q_{f}$ for fresh water and $Q_{s}$ for salt water) we formulate the problem in terms of the stream function in an axial symmetric flow domain in $\mathbb{R}^{n}(n=2,3)$. We prove the existence of a continuous free boundary which ends up in the well, located on the central axis. Moreover, we show that the free boundary has a tangent at the well and approaches it in a $C^{1}$ sense. Using the method of separation of variables we also give a result concerning the asymptotic behaviour of the free boundary at the well. For a given total discharge $\left(Q:=Q_{f}+Q_{s}\right)$ we consider the vanishing $Q_{s}$ limit. We show that a free boundary arises with a cusp at the central axis, having a positive distance from the well. This work is a continuation of $[5,6]$.
\end{abstract}

Keywords: Porous media flow; free boundary problem.

\section{Introduction}

In two previous papers [5, 6], we studied a free boundary problem that results from a model describing the withdrawal of fluid from a reservoir. In that model we considered the stationary flow of two incompressible fluids through a homogeneous and isotropic porous medium (the reservoir). The fluids have constant but different densities and are assumed to be separated by an abrupt transition, an interface. In the reservoir one or more wells are present to recover one of the fluids. Such models are relevant, for instance, when designing freshwater reservoirs in coastal regions. Then fresh water overlays salt water from the sea.

When we think of a horizontal interface in the absence of the wells, we will observe an upconed interface after applying the wells to the fluid on top, see Fig. 1, where a reservoir with only one well is shown.

Assuming only horizontal flow along the vertical boundaries of the reservoir, with a fixed and prescribed position of the interface $\left(u_{0}\right)$, a stationary flow and a stationary interface may result for which the fluid below is stagnant. The fluid on top is drawn into the wells. This case is studied in detail in [5]. It leads to an elliptic free boundary problem involving a parameter $Q$, which is related to the withdrawal rate or discharge of the wells. It was shown that a critical rate $Q_{c r}>0$ exists such that for $Q<Q_{c r}$ the interface can be represented by an analytic function of the horizontal coordinates. Moreover, the height of the interface increases whenever the rate increases. At $Q=$ $Q_{c r}$ a cusp develops in the interface, being still at a positive distance from the well, see Fig. 2 . These results were proven for flows in $\mathbb{R}^{n}, n \geqslant 2$.

$\dagger$ Author to whom correspondence should be addressed. 


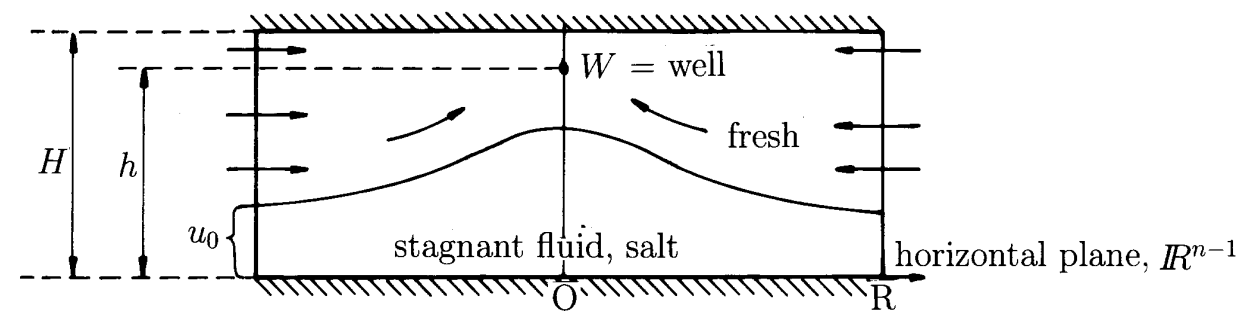

FIG. 1. Smooth upconed interface, $Q<Q_{c r}$.

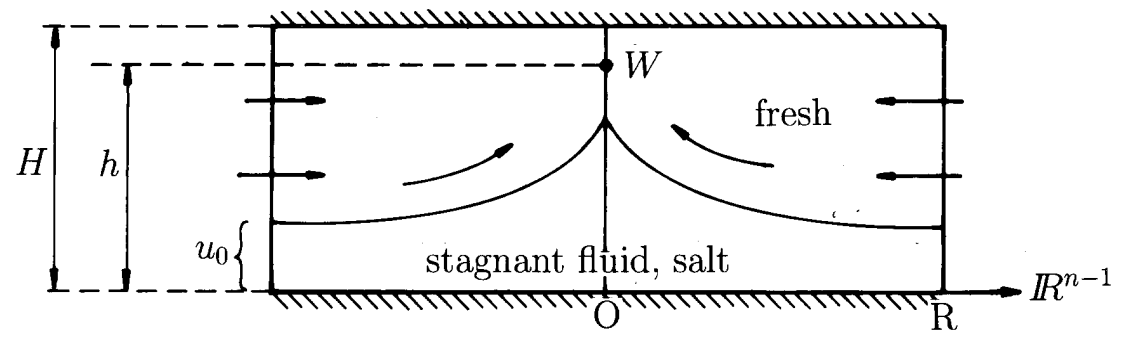

FIG. 2. Interface with cusp, $Q=Q_{c r}$.

In the second work [6], we analysed in detail the local behaviour near the cusp. This was performed for the two-dimensional case $(n=2)$ only. Applying our local results to a configuration with one well, as in Fig. 2, we obtain for points $(x, z)$ on the interface ( $x$ horizontal, $z$ vertical) near the cusp $\left(x_{0}, z_{0}\right)$

$$
\lim _{x \rightarrow x_{0}} \frac{\left|x-x_{0}\right|}{\left(z_{0}-z\right)^{3 / 2}}=C
$$

for some constant $C>0$.

Keeping the reservoir dimensions $(H$ and $R$ ) and all physical parameters fixed, the value of the critical rate $Q_{c r}$ only depends on $h-u_{0}$, where $h$ denotes the distance between the well and the bottom of the reservoir. We conjecture that $Q_{c r}=Q_{c r}\left(h-u_{0}\right)$ is continuous and strictly increasing with $Q_{c r}(0)=0$.

Instead of considering the critical cusp case as the limit of subcritical cases, all having smooth interfaces with stagnant salt water below, we propose in this paper a different stategy. In this strategy we let the salt water move as well and we characterize the cusp case as the limit of vanishing flow in the saltwater region.

Thus we first need to address the problem of what happens when the salt water also moves towards the well. We expect a fluid distribution as in Fig. 4.

The main result of this paper is that there are stationary solutions of this type. We study these 


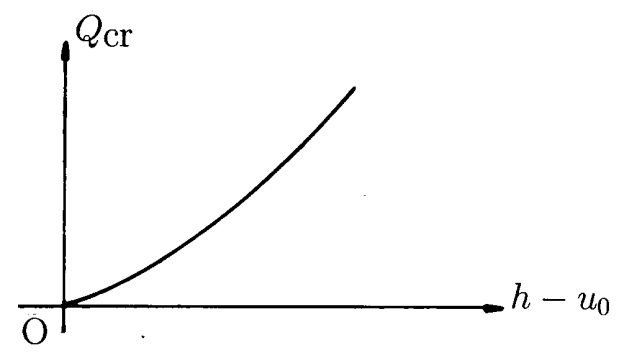

FIG. 3. Sketch of the behaviour of $Q_{c r}$; the shape of the curve is unknown.

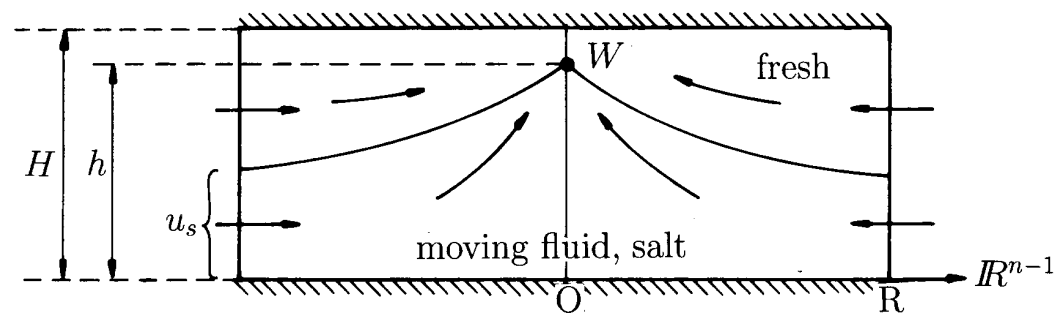

FIG. 4. Salt water moves towards the well.

solutions for axial symmetric flows $(n=2,3)$, with only one well on the central axis. This allows us to formulate the problem in terms of a stream function, as in [2] or [4].

In Section 2 we present the weak formulation for the flow problem in a bounded, axial symmetric reservoir of constant thickness. Now the formulation involves two parameters: $Q_{s}$ (outflow of salt water at the well) and $Q_{f}$ (outflow of fresh water at the well), or rather $Q_{s}$ and $Q:=Q_{s}+Q_{f}$ (total outflow at the well). As a result of this formulation we are able to prove that a weak solution exists (Section 3). In Section 4 we show that the interface is a continuous curve in the $z$-direction. On the axis it ends up in the well $W$ and on the lateral boundary in a well-defined point $\left(R, u_{S}\right)$.

In Sections 5 and 6 we consider the free boundary near the well. First, we show in Section 5 that the free boundary has a tangent at $W$ and approaches it in a $C^{1}$-sense. The tangent direction is given by the angle

$$
\omega_{*}= \begin{cases}\frac{\pi}{2} \frac{Q_{s}-Q_{f}}{Q_{s}+Q_{f}} & \text { for } n=2, \\ \arcsin \frac{Q_{s}-Q_{f}}{Q_{s}+Q_{f}} & \text { for } n=3,\end{cases}
$$

with respect to the horizontal plane. Note that $\omega_{*}$ only depends on the discharge at the well and does not involve density (gravity) effects. The asympotic behaviour at $W$ is studied in Section 6 . 
Introducing polar coordinates and writing, for free boundary points $(r, z)$,

$$
r+i(z-h)=e^{s+i \omega(s)},
$$

we give by means of the method of separation of variables an estimate for the rate of convergence (see Theorem 6.7)

$$
\omega(s) \rightarrow \omega_{*} \text { as } s \searrow-\infty .
$$

Concerning the vanishing $Q_{s}$ limit we only have a partial result. In Section 7 we show that if $Q$ is sufficiently small, depending only on the reservoir dimensions and the position of the well, then $Q_{s} \searrow 0$ results in an interface with a cusp at the origin, having a positive distance from the well, and with stagnant fluid below it. For larger values of $Q$ we have no precise mathematical results when taking this limit. However, we conjecture the following behaviour: if $Q \leqslant Q_{c r}(h)$, see Fig. 3, then $Q_{s} \searrow 0$ results in a decreasing sequence of interfaces, converging to a cusped interface satisfying $Q_{c r}\left(h-u_{0}\right)=Q$, where $u_{0}=\lim _{Q_{s} \searrow 0} u_{s}$. Only for $Q<Q_{c r}(h)$ is this rigorously demonstrated.

If $Q>Q_{c r}(h)$, then $Q_{s} \searrow 0$ results in a decreasing sequence of interfaces converging to a cusped interface which partly coincides with the horizontal bottom of the reservoir, see Fig. 5.

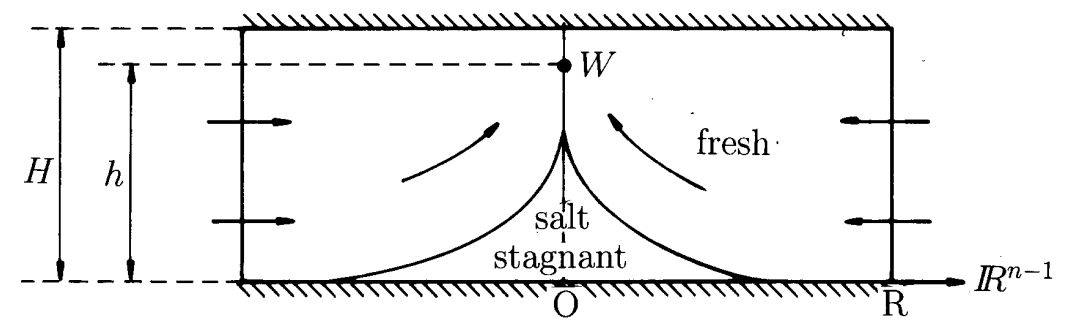

FIG. 5. Conjectured interface for $Q>Q_{c r}(h)$.

In the two-dimensional setting such interfaces have been constructed explicitly using hodograph techniques, for example, see [12].

\section{Formulation of the problem}

Let the reservoir occupy the bounded, axial-symmetric region

$$
\tilde{\Omega}=\left\{\left(x_{1}, \ldots, x_{n}\right): \sqrt{x_{1}^{2}+\cdots+x_{n-1}^{2}}<R, 0<x_{n}<H\right\},
$$

with $n=2,3$ describing the physical cases. It is saturated by either fresh water or salt water, which are macroscopically separated by an interface $S$. We also write

$$
\tilde{\Omega}=\tilde{\Omega}_{f} \cup S \cup \tilde{\Omega}_{s},
$$

where $\tilde{\Omega}_{f}$ and $\tilde{\Omega}_{s}$ denote the regions filled up by the fresh and salt groundwater, see Fig. 6 . 


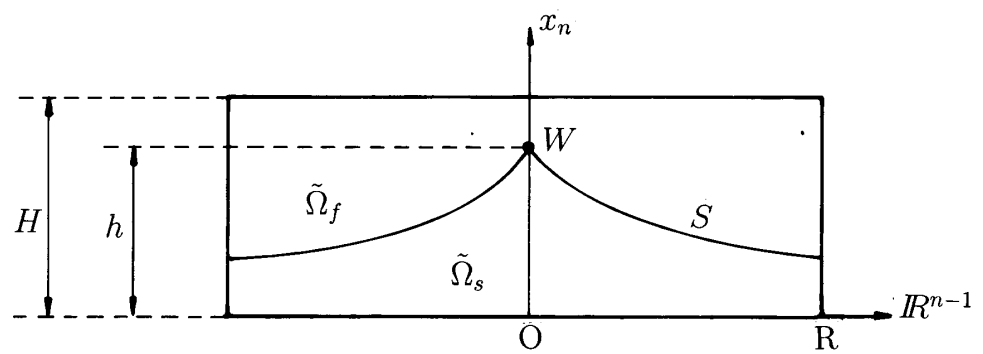

FIG. 6. Axial symmetric reservoir.

At the central axis, a well $W$ is located at a distance $h \in] 0, H[$ above the horizontal plane $\left\{x_{n}=0\right\}$. We will study the case where both fresh and salt water are being extracted from the reservoir through $W$. Let $Q_{f}>0$ denote the discharge of fresh water and $Q_{s}>0$ the discharge of salt water. Then $Q:=Q_{s}+Q_{f}$ is the total production rate of fluid from the reservoir. Each fluid has a constant specific weight $\gamma_{i}$, with $0<\gamma_{f}<\gamma_{s}<\infty$ and the fluid-medium interaction is characterized by a constant mobility $\lambda>0$. The model is described by Darcy's law

$$
q+\lambda\left(\operatorname{grad} p+\gamma e_{z}\right)=0
$$

and the fluid balance equation

$$
\operatorname{div} q=-Q \delta_{W}
$$

in $\tilde{\Omega}$, where $\gamma=\gamma_{f}$ in $\tilde{\Omega}_{f}$ and $\gamma=\gamma_{s}$ in $\tilde{\Omega}_{s}$. In these equations, $q$ denotes the specific discharge, $p$ the pressure, $e_{z}$ the unit vector in the positive $x_{n}$-direction (against the direction of gravity) and $\delta_{W}$ the Dirac distribution at the point $W$. Along the upper and lower boundary of $\tilde{\Omega}$ we require a no-flow condition, expressed by

$$
q \cdot e_{z}=0 \quad \text { on } \quad\left\{x_{n}=0\right\} \cup\left\{x_{n}=H\right\} .
$$

Along the cylindrical, lateral boundary we assume horizontal flow, i.e.

$$
q \text { is normal at }\left\{x_{1}^{2}+\cdots+x_{n-1}^{2}=R^{2}\right\} .
$$

Because of the cylindrical form of the reservoir and the central location of the well, we expect axial symmetry of the unknowns. Thus introducing

$$
r=\sqrt{x_{1}^{2}+\cdots+x_{n-1}^{2}} \text { and } z=x_{n}
$$

we consider $p, q$ and $\gamma$ to be functions of these variables. We obtain in the two-dimensional domain

$$
\Omega=\{(r, z): 0<r<R, 0<z<H\}
$$

Darcy's law again

$$
q+\lambda\left(\nabla p+\gamma e_{z}\right)=0
$$


where now $q=q_{r} e_{r}+q_{z} e_{z}$, with $e_{r}=\frac{1}{r}\left(x_{1}, \ldots, x_{n-1}, 0\right)$ and $e_{z}=(0, \ldots, 0,1)$, and $\nabla=\left(\partial_{r}, \partial_{z}\right)$. The fluid balance equation in $\Omega$ becomes

$$
\frac{1}{r^{n-2}} \partial_{r}\left(r^{n-2} q_{r}\right)+\partial_{z} q_{z}=0
$$

The latter equation suggests the introduction of a stream function $\psi: \Omega \rightarrow \mathbb{R}$ satisfying

$$
q=\left(-\frac{1}{r^{n-2}} \partial_{z} \psi, \frac{1}{r^{n-2}} \partial_{r} \psi\right) .
$$

At this point we first introduce dimensionless variables. Let $\Gamma=\lambda\left(\gamma_{s}-\gamma_{f}\right)$. Then we normalize

$$
\begin{aligned}
\psi & :=\psi /\left(\Gamma H^{n-2}\right) ; Q, Q_{s} \text { and } Q_{f} \text { similar } \\
\gamma & :=\left(\gamma-\gamma_{f}\right) /\left(\gamma_{s}-\gamma_{f}\right) \\
r & =r / H ; z, H, R \text { and } h \text { similar. }
\end{aligned}
$$

An equation for $\psi$ results by taking the two-dimensional curl of Darcy's law (2.1) and by substituting (2.2) into the result, see also [2] or [4]. This yields

$$
\nabla \cdot\left(\frac{1}{r^{n-2}} \nabla \psi+\gamma e_{r}\right)=0 \quad \text { in } \Omega,
$$

with

$$
\gamma=\left\{\begin{array}{lll}
0 & \text { in } & \Omega_{f}, \\
1 & \text { in } & \Omega_{s},
\end{array}\right.
$$

where $\Omega_{f}$ and $\Omega_{s}$ now denote the subregions of $\Omega$ filled up by fresh and salt water, see Fig. 7 .

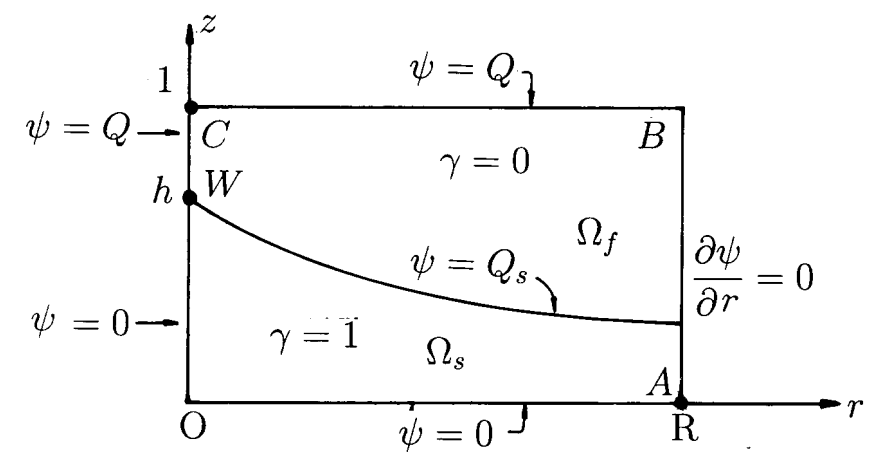

FIG. 7. Boundary conditions for $\psi$.

Because the top and bottom of the reservoir are impervious, the stream function must be constant there. The same is true on the symmetry axis, except, of course, at the location $(0, h)$ of the well, 
where fluid is being extracted from the reservoir. There the stream function exhibits a jump $Q$. With reference to Fig. 7 we take

$$
\psi=0 \quad \text { along } \quad A O W
$$

and

$$
\psi=Q \quad \text { along } B C W .
$$

The assumption of only horizontal flow along the lateral boundary requires

$$
\partial_{r} \psi=0 \text { along } A B .
$$

For future reference, we denote the boundary of $\Omega$ by $\partial \Omega$, the part $A O C B$ by $\partial_{D} \Omega$ and the part $A B$ by $\partial_{N} \Omega$. Because the interface is stationary, $\psi$ must be constant there as well. To ensure that the prescribed saltwater discharge $Q_{s}$ is being extracted from the reservoir we take

$$
\psi=Q_{s} \quad \text { along the fresh-salt interface. }
$$

Now considering (2.3) in $\Omega_{f}$ and $\Omega_{s}$, we expect to find, by the strong maximum principle,

$$
0<\psi<Q_{s} \text { in } \Omega_{s}
$$

and

$$
Q_{s}<\psi<Q \quad \text { in } \Omega_{f} .
$$

Therefore we write

$$
\gamma=1-H\left(\psi-Q_{s}\right) \text { in } \Omega,
$$

where $H(\cdot)$ denotes the Heaviside graph

$$
H(s)= \begin{cases}1 & \text { for } s>0 \\ {[0,1]} & \text { for } s=0 \\ 0 & \text { for } s<0\end{cases}
$$

We expect certain smoothness (at least Lipschitz continuity) away from the location of the well. To capture the singular behaviour of $\psi$ there, we consider the function $\psi_{0}$ which corresponds to $\gamma=0$ in $\Omega$ : i.e. it satisfies

$$
\nabla \cdot\left(\frac{1}{r^{n-2}} \nabla \psi_{0}\right)=0 \text { in } \Omega
$$

and the $\psi$-boundary conditions on $\partial \Omega$.

LEMMA 2.1 There exists exactly one such $\psi_{0}$, smooth (at least $C^{1}$ ) away from $W$, with $\partial_{z} \psi_{0}>0$ in $\Omega$. Near $W$ it satisfies

$$
\psi_{0}=\psi_{*}+\text { smooth terms, }
$$

where

$$
\psi_{*}(r, z):= \begin{cases}\frac{Q}{2}\left(\frac{2}{\pi} \arctan \frac{z-h}{r}+1\right) & \text { for } n=2, \\ \frac{Q}{2}\left(\frac{z-h}{\left(r^{2}+(z-h)^{2}\right)^{1 / 2}}+1\right) & \text { for } n=3 .\end{cases}
$$


Proof. We give the proof only for $n=3$. It uses the pressure formulation in the three-dimensional domain $\tilde{\Omega}$. Let $\tilde{p}_{0}$ be the weak solution of the problem

$$
\begin{cases}\Delta \tilde{p}_{0}=2 \pi Q \delta_{W} & \text { in } \tilde{\Omega}, \\ \partial_{z} \tilde{p}_{0}=0 & \text { on top and bottom of } \tilde{\Omega} \\ \tilde{p}_{0}=0 & \text { on lateral side of } \tilde{\Omega} .\end{cases}
$$

By standard elliptic theory, e.g. see [9], there exists a unique $\tilde{p}_{0}$ which is smooth outside $W$. Clearly $\tilde{p}_{0}$ is axially symmetric. Therefore, writing $x=\left(x_{1}, x_{2}, x_{3}\right)$,

$$
\tilde{p}_{0}(x)=p_{0}(r, z), \quad \text { with }(r, z) \in \Omega \text {. }
$$

The function $p_{0}$ is smooth inside $\Omega$ where it satisfies the equation

$$
\partial_{r}\left(r \partial_{r} p_{0}\right)+\partial_{z}\left(r \partial_{z} p_{0}\right)=0
$$

Since $\Omega$ is simply connected, this implies the existence of a unique (up to an additive constant) function $\psi_{0}: \bar{\Omega} \backslash W \rightarrow \mathbb{R}$ which satisfies

$$
\partial_{z} p_{0}=-\frac{1}{r} \partial_{r} \psi_{0} \quad \text { and } \quad \partial_{r} p_{0}=\frac{1}{r} \partial_{z} \psi_{0}
$$

One easily verifies that $\psi_{0}$ solves (2.4) in $\Omega$, that $\psi_{0}$ is piecewise constant on $\partial_{D} \Omega$ (except at $W$ ) and that $\partial_{r} \psi_{0}=0$ on $\partial_{N} \Omega$. To show that $\psi_{0}$ jumps with $Q$ at $W$ we integrate the three-dimensional equation for $\tilde{p}_{0}$ over a small cylindrical neighbourhood of $W$. For $\varepsilon, \delta>0$ and sufficiently small, let

$$
C_{\delta}^{\varepsilon}:=\left\{x=\left(x_{1}, x_{2}, x_{3}\right): \sqrt{x_{1}^{2}+x_{2}^{2}}<\varepsilon,\left|x_{3}-h\right|<\delta\right\} .
$$

Then

$$
\begin{aligned}
2 \pi Q & =\int_{\partial C_{s}^{\varepsilon}} \operatorname{grad} \tilde{p}_{0} \cdot v \\
& =2 \pi \int_{h-\delta}^{h+\delta} \varepsilon \partial_{r} p_{0}(\varepsilon, z) \mathrm{d} z+\int_{\left\{\sqrt{x_{1}^{2}+x_{2}^{2}}<\varepsilon\right\}}\left\{\partial_{z} \tilde{p}_{0}\left(x_{1}, x_{2}, \delta\right)-\partial_{z} \tilde{p}_{0}\left(x_{1}, x_{2},-\delta\right)\right\} \mathrm{d} x_{1} \mathrm{~d} x_{2} .
\end{aligned}
$$

In the first integral we replace the integrand by $\partial_{z} \psi_{0}(\varepsilon, z)$.

Then for $\delta$ fixed and $\varepsilon \searrow 0$ we find

$$
Q=\psi_{0}(0, h+\delta)-\psi_{0}(0, h-\delta),
$$

which shows that $\psi_{0}$ indeed satisfies the correct jump condition at $W$. The proof concerning the $z$-monotonicity of $\psi_{0}$ in $\Omega$ follows as a special case of the proof of Proposition 3.4. (i.e. without gravity). It will therefore not be given here. 
The asymptotic expressions follow from the observation that near $W, \tilde{p}_{0}$ can be written as

$$
\tilde{p}_{0}(x)=-2 \pi Q F(x-W)+\text { smooth terms, }
$$

where $F$ is the fundamental solution of $-\Delta$ with respect to the origin. Consequently, near $W$,

$$
\operatorname{grad} \tilde{p}_{0}(x)=\frac{Q}{2} \frac{x-W}{|x-W|^{3}}+\text { smooth terms }
$$

or

$$
\nabla p_{0}(r, z)=\frac{Q}{2\left(r^{2}+(z-h)^{2}\right)^{3 / 2}}\left(r e_{r}+(z-h) e_{z}\right)+\text { smooth terms. }
$$

Finally, we use relations (2.5) and obtain

$$
\psi_{0}=\psi_{*}+\text { smooth terms near } W
$$

with $\psi_{*}$ as in the assertion.

Using $\psi_{0}$ we introduce the following weak formulation. Let

$$
V=\left\{\zeta \in H^{1,2}(\Omega): \zeta=0 \text { in } \partial_{D} \Omega \text { and } r^{\frac{2-n}{2}} \nabla \zeta \in L^{2}\left(\Omega ; \mathbb{R}^{2}\right)\right\} .
$$

Find $\psi \in \psi_{0}+V, \gamma \in L^{\infty}(\Omega)$ and $\gamma_{N} \in L^{\infty}\left(\partial_{N} \Omega\right)$ such that

$$
\int_{\Omega} \nabla \zeta \cdot\left\{\frac{1}{r^{n-2}} \nabla\left(\psi-\psi_{0}\right)+\gamma e_{r}\right\}=\int_{\partial_{N} \Omega} \zeta \gamma_{N}
$$

for all $\zeta \in V$, and

$$
\begin{cases}\gamma \in 1-H\left(\psi-Q_{S}\right) & \text { in } \quad \Omega \\ \gamma_{N} \in 1-H\left(\psi-Q_{S}\right) & \text { in } \partial_{N} \Omega .\end{cases}
$$

REMARK. If the value of $\gamma$ would exist at $\partial_{N} \Omega$, and coincide with $\gamma_{N}$, then the weak formulation (at least formally) implies $\partial_{r}\left(\psi-\psi_{0}\right)=0$ at $\partial_{N} \Omega$. Since $\partial_{r} \psi_{0}=0$ along $\partial_{N} \Omega$, this gives the desired boundary condition for $\psi$.

\section{Existence of weak solution}

LEMMA 3.1 There exists at least one weak solution $\left\{\psi, \gamma, \gamma_{N}\right\}$.

Proof. In the equation for $\psi$ we introduce an $\varepsilon$-regularization with respect to $\gamma$ and, based on the function $\psi_{0}$ in Section 2, an $\varepsilon$-regularization with respect to the term $1 / r^{n-2}$ in the differential equation, the Dirichlet condition on the axis, and due to the special construction below also with respect to the domain.

The perturbed domain is

$$
\left.\Omega_{\varepsilon}:=\right] 0, R-\varepsilon[\times] 0,1[
$$

and the perturbed function

$$
\psi_{0, \varepsilon}: \bar{\Omega}_{\varepsilon} \rightarrow \mathbb{R}
$$


is defined by the shift

$$
\psi_{0, \varepsilon}(r, z)=\psi_{0}(r+\varepsilon, z) \quad \text { for }(r, z) \in \bar{\Omega}_{\varepsilon} .
$$

Each function $\psi_{0, \varepsilon}$ is a smooth solution of the perturbed equation

$$
\nabla \cdot\left(\frac{1}{(r+\varepsilon)^{n-2}} \nabla \psi_{0, \varepsilon}\right)=0 \text { in } \Omega_{\varepsilon}
$$

and satisfies the boundary conditions, see Fig. 8,

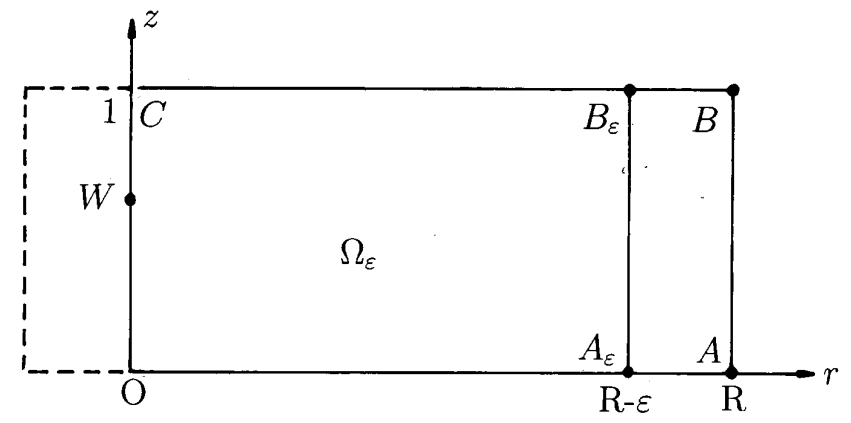

mbox

FIG. 8. Shifted domain $\Omega_{\varepsilon}$.

$$
B C_{\varepsilon}\left\{\begin{array}{l}
\psi_{0, \varepsilon}=0 \text { on } O A_{\varepsilon}, \quad \psi_{0, \varepsilon}=Q \text { on } B_{\varepsilon} C \\
\partial_{r} \psi_{0, \varepsilon}=0 \text { on } A_{\varepsilon} B_{\varepsilon} \\
\psi_{0, \varepsilon}=\psi_{0}(\varepsilon, \cdot) \text { on } O C,
\end{array}\right.
$$

where $\psi_{0}(\varepsilon, \cdot)$ satisfies : $\psi_{0}(\varepsilon, 0)=0, \psi_{0}(\varepsilon, 1)=Q, \partial_{z} \psi_{0}(\varepsilon, \cdot)>0$ and $\psi_{0}(\varepsilon, z) \rightarrow \psi_{0}(0, z)$ as $\varepsilon \searrow 0$ pointwise for $z \neq h$ (with $\psi_{0}(0, \cdot)=0$ on $O W$ and $\psi_{0}(0, \cdot)=Q$ on $W C$ ), see Lemma 2.1.

Next we turn to the $\varepsilon$-regularization for $\psi$, which we define through the problems (for any small $\varepsilon>0)$

$$
P_{\varepsilon}\left\{\begin{array}{l}
\nabla \cdot\left(\frac{1}{(r+\varepsilon)^{n-2}} \nabla \psi+\left(1-H_{\varepsilon}\left(\psi-Q_{s}\right)\right) e_{r}\right)=0 \quad \text { in } \quad \Omega_{\varepsilon}, \\
\psi \text { satisfies } B C_{\varepsilon},
\end{array}\right.
$$

where $H_{\varepsilon}$ is a smooth monotone approximation of the Heaviside graph. As for instance in [1] or [7], Problem $P_{\varepsilon}$ has a unique smooth solution $\psi_{\varepsilon}$. We first show that Problem $P_{\varepsilon}$ satisfies a comparison principle.

PROPOSITION 3.2 Let $\psi_{1}$ and $\psi_{2}$ be two solutions of the $\psi_{\varepsilon}$-equation satisfying $\psi_{1} \leqslant \psi_{2}$ on $\partial_{D} \Omega_{\varepsilon}$ and $\partial_{r}\left(\psi_{1}-\psi_{2}\right)=0$ on $\partial_{N} \Omega_{\varepsilon}$. Then

$$
\psi_{1} \leqslant \psi_{2} \text { in } \Omega_{\varepsilon}
$$


Proof. The proof is a modification of the proof of [11: Theorem 1]. There he tests the equation for the difference $\psi_{1}-\psi_{2}$ with the function (for $\delta>0$ )

$$
\zeta=\frac{w}{\delta+w} \quad \text { with } w=\left(\psi_{1}-\psi_{2}-\delta\right)_{+} .
$$

Following this procedure we arrive at the identity

$$
\begin{gathered}
\delta \int_{\left\{\psi_{1}-\psi_{2} \geqslant \delta\right\}} \frac{1}{(r+\varepsilon)^{n-2}} \frac{|\nabla w|^{2}}{(\delta+w)^{2}}+\delta \int_{\left\{\psi_{1}-\psi_{2} \geqslant \delta\right\}}\left(H_{2}-H_{1}\right) \frac{\partial_{r} w}{(\delta+w)^{2}} \\
+\int_{\left\{\psi_{1}-\psi_{2} \geqslant \delta\right\} \cap\{r=R-\varepsilon\}}\left(H_{1}-H_{2}\right) \frac{w}{\delta+w}=0,
\end{gathered}
$$

where we used the notation $H_{i}=H_{\varepsilon}\left(\psi_{i}-Q_{s}\right)$. The first and second term are as in [11] and can be treated similarly. The third term is non-negative, by the monotonicity of $H_{\varepsilon}$, and can therefore be disregarded from the estimates. Proceeding as in [11] results in $\psi_{1} \leqslant \psi_{2}$ in $\Omega$.

Corollary $3.30 \leqslant \psi_{\varepsilon} \leqslant Q$ in $\Omega_{\varepsilon}$.

Proof. Since constants satisfy the equation, these inequalities follow from $B C_{\varepsilon}$.

Returning to the existence proof we introduce the difference

$$
v_{\varepsilon}:=\psi_{\varepsilon}-\psi_{0, \varepsilon} \text { in } \Omega_{\varepsilon},
$$

which satisfies the equation, with $h_{\varepsilon}:=H_{\varepsilon}\left(v_{\varepsilon}+\psi_{0, \varepsilon}-Q_{s}\right)$,

$$
\nabla \cdot\left(\frac{1}{(r+\varepsilon)^{n-2}} \nabla v_{\varepsilon}+\left(1-h_{\varepsilon}\right) e_{r}\right)=0 \quad \text { in } \quad \Omega_{\varepsilon}
$$

and the homogeneous boundary conditions

$$
\left.v_{\varepsilon}\right|_{\partial_{D} \Omega_{\varepsilon}}=0 \text { and }\left.\partial_{r} v_{\varepsilon}\right|_{\partial_{N} \Omega_{\varepsilon}}=0
$$

Multiplying the equation by $v_{\varepsilon}$ and integrating over $\Omega_{\varepsilon}$ gives

$$
\int_{\Omega_{\varepsilon}} \frac{1}{(r+\varepsilon)^{n-2}}\left|\nabla v_{\varepsilon}\right|^{2}=-\int_{\Omega_{\varepsilon}}\left(1-h_{\varepsilon}\right) \partial_{r} v_{\varepsilon}+\int_{\{r=R-\varepsilon\}}\left(1-h_{\varepsilon}\right) v_{\varepsilon} .
$$

Absorbing the first term in the right-hand side and using Corrollary 3.3. for the second term, we obtain the uniform estimate

$$
\int_{\Omega_{\varepsilon}} \frac{1}{(r+\varepsilon)^{n-2}}\left|\nabla v_{\varepsilon}\right|^{2} \leqslant C .
$$

Introducing the characteristic function of the set $\Omega_{\varepsilon}$, we deduce

$$
\chi_{\Omega_{\varepsilon}} \frac{\nabla v_{\varepsilon}}{(r+\varepsilon)^{\frac{n}{2}-1}} \text { is uniformly bounded in } L^{2}\left(\mathbb{R}^{2} ; \mathbb{R}^{2}\right),
$$


$\chi_{\Omega_{\varepsilon}} v_{\varepsilon}$ is uniformly bounded in $L^{\infty}\left(\mathbb{R}^{2}\right)$.

Consequently, there exist functions $v^{*} \in L^{2}\left(\Omega ; \mathbb{R}^{2}\right)$ and $v \in L^{2}(\Omega)$ such that for a sequence $\varepsilon \searrow 0$

$$
\begin{gathered}
\chi_{\Omega_{\varepsilon}} \frac{\nabla v_{\varepsilon}}{(r+\varepsilon)^{\frac{n}{2}-1}} \rightarrow \chi_{\Omega} v^{*} \text { weakly in } L^{2}\left(\mathbb{R}^{2} ; \mathbb{R}^{2}\right), \\
\chi_{\Omega_{\varepsilon}} v_{\varepsilon} \rightarrow \chi_{\Omega} v \text { weak star in } L^{\infty}\left(\mathbb{R}^{2}\right) .
\end{gathered}
$$

Since then $\chi_{\Omega_{\varepsilon}} \nabla v_{\varepsilon} \rightarrow \chi_{\Omega} r^{\frac{n}{2}-1} v^{*}$ weakly in $L^{2}\left(\mathbb{R}^{2} ; \mathbb{R}^{2}\right)$, it follows that $v \in H^{1,2}(\Omega)$ with

$$
\nabla v=r^{\frac{n}{2}-1} v^{*} \quad \text { a.e. in } \Omega .
$$

Therefore $v_{\varepsilon} \rightarrow v$ weakly in $H_{l o c}^{1.2}(\Omega)$ and thus for a subsequence

$$
v_{\varepsilon} \rightarrow v \quad \text { a.e. in } \Omega \text {. }
$$

Since also $\psi_{0, \varepsilon} \rightarrow \psi$ locally uniformly in $\bar{\Omega} \backslash W$, there exists $\hat{\gamma} \in L^{\infty}(\Omega)$ such that

$$
\chi_{\Omega_{\varepsilon}} H_{\varepsilon}\left(v_{\varepsilon}+\psi_{0, \varepsilon}-Q_{s}\right) \rightarrow \hat{\gamma}
$$

weak star in $L^{\infty}\left(\mathbb{R}^{2}\right)$ with $\hat{\gamma} \in H\left(v+\psi_{0}-Q_{s}\right)$, and $\hat{\gamma}_{N} \in L^{\infty}\left(\partial_{N} \Omega\right)$ such that

$$
H_{\varepsilon}\left(v_{\varepsilon}+\psi_{0, \varepsilon}-Q_{s}\right)(R-\varepsilon, \cdot) \rightarrow \hat{\gamma}_{N}(R, \cdot)
$$

weak star in $L^{\infty}(] 0,1[)$ with $\hat{\gamma}_{N} \in H\left(v+\psi_{0}-Q_{s}\right)$.

Finally, we test the $v_{\varepsilon}$-equation with $\zeta \in V$. This gives

$$
\begin{gathered}
\int_{\mathbb{R}^{2}} \frac{\nabla \zeta}{(r+\varepsilon)^{\frac{n}{2}-1}} \cdot\left(\chi_{\Omega_{\varepsilon}} \frac{\nabla v_{\varepsilon}}{(r+\varepsilon)^{\frac{n}{2}-1}}\right)+\int_{\mathbb{R}^{2}} \partial_{r} \zeta \chi_{\Omega_{\varepsilon}}\left(1-H_{\varepsilon}\left(v_{\varepsilon}+\psi_{0, \varepsilon}-Q_{s}\right)\right) \\
=\int_{\{r=R-\varepsilon\}} \zeta\left(1-H_{\varepsilon}\left(v_{\varepsilon}+\psi_{0, \varepsilon}-Q_{s}\right)\right) .
\end{gathered}
$$

We now have all ingredients to pass to the limit for $\varepsilon \searrow 0$, which gives the weak equation ( $\star$ ) for $\psi:=v+\psi_{0}$.

Crucial for the existence of a free boundary is the inequality.

PROPOSITION $3.4 \quad \partial_{z} \psi \geqslant 0$ in sense of distributions.

Proof. For $\delta>0$, sufficiently small, we define the domain

$$
\left.\Omega_{\varepsilon}^{\delta}=\right] 0, R-\varepsilon[\times] \delta, 1[
$$

and the translated function

$$
\psi_{\varepsilon}^{\delta}(r, z)=\psi_{\varepsilon}(r, z-\delta) \quad \text { for }(r, z) \in \Omega_{\varepsilon}^{\delta} .
$$

Using the properties of $B C_{\varepsilon}$ and Corrollary 3.3 we have

$$
\psi_{\varepsilon}^{\delta} \leqslant \psi_{\varepsilon} \text { on } \partial_{D} \Omega_{\varepsilon}^{\delta} \text { and } \partial_{r}\left(\psi_{\varepsilon}^{\delta}-\psi_{\varepsilon}\right)=0 \text { on } \partial_{N} \Omega_{\varepsilon}^{\delta} .
$$

Since $\psi_{\varepsilon}^{\delta}$ satisfies the $\psi_{\varepsilon}$-equation as well, the comparison principle gives $\psi_{\varepsilon}^{\delta} \leqslant \psi_{\varepsilon}$ in $\Omega_{\varepsilon}^{\delta}$. From this inequality $\partial_{z} \psi_{\varepsilon} \geqslant 0$ in $\Omega_{\varepsilon}$ is immediate. Letting $\varepsilon \searrow 0$ completes the proof. 
For later use we show continuity properties of the solution $\psi$.

THEOREM $3.5 \psi$ is Hölder continuous in $\bar{\Omega} \backslash W$.

Proof. The Hölder continuity away from the axis follows from standard techniques. Since $\gamma_{N}$ depends only on the $z$-variable, the weak equation for $\psi$ can be written as

$$
\int_{\Omega} \nabla \zeta \cdot\left(\frac{1}{r^{n-2}} \nabla \psi+\left(\gamma-\gamma_{N}\right) e_{r}\right)=0
$$

for all $\zeta \in V$, with $\zeta(r, z)=0$ for small $r$. If

$$
\zeta=\eta^{2}(\psi-m) \quad \text { with } m \in \mathbb{R}, \eta \in C_{0}^{\infty}\left(\mathbb{R}^{2}\right)
$$

is such a test function, we derive that

$$
\int_{\Omega} \frac{\eta^{2}}{r^{n-2}}|\nabla \psi|^{2} \leqslant C \int_{\Omega}\left(\eta^{2} r^{n-2}+\frac{|\nabla \eta|^{2}}{r^{n-2}}(\psi-m)^{2}\right) .
$$

In particular, if $x_{0}=\left(r_{0}, z_{0}\right) \in \bar{\Omega}$ with $r_{0}>\delta(\delta>0$, fixed $)$ and if $r_{0}-2 \rho \geqslant \delta$, let $\eta$ be a standard cut-off function satisfying $\eta(x)=1$ for $\left|x-x_{0}\right| \leqslant \rho$ and $\eta(x)=0$ for $\left|x-x_{0}\right| \geqslant 2 \rho$. In the three cases:

$$
\begin{aligned}
& \text { (i) } \quad B_{2 \rho}\left(x_{0}\right) \subset \Omega, m=\underset{\Omega \cap B_{2 \rho}\left(x_{0}\right) \backslash B_{\rho}\left(x_{0}\right)}{\int} \psi ; \\
& \text { (ii) } \quad r_{0}=R,\left[z_{0}-2 \rho, z_{0}+2 \rho\right] \subset[0,1], m \text { as in (i); } \\
& \text { (iii) } \left.z_{0}=0 \text { (or } 1\right), 2 \rho<1, m=0 \text { (or } Q \text { ); }
\end{aligned}
$$

we can apply the above inequality. Using Poincaré's inequality for $\psi-m$ on $\Omega \cap B_{2 \rho}\left(x_{0}\right) \backslash B_{\rho}\left(x_{0}\right)$ we obtain an estimate

$$
\int_{\Omega \cap B_{\rho}\left(x_{0}\right)}|\nabla \psi|^{2} \leqslant C_{\delta}\left(\rho^{2}+\int_{\Omega \cap B_{2 \rho}\left(x_{0}\right) \backslash B_{\rho}\left(x_{0}\right)}|\nabla \psi|^{2}\right) .
$$

From this we deduce for given $\delta>0$ (as above) and $\varepsilon>0$

$$
\int_{\Omega \cap B_{\rho}\left(x_{0}\right)}|\nabla \psi|^{2} \leqslant C_{\delta, \varepsilon} \rho^{2-\varepsilon}
$$

for all $x_{0}=\left(r_{0}, x_{0}\right) \in \bar{\Omega}$ and $\rho>0$ with $r_{0}-2 \rho \geqslant \delta$. Then by the Morrey lemma, see [9], the Hölder continuity of $\psi$ away from the axis and with any Hölder exponent follows.

For $n=2$, the same procedure applies at the axis outside the well. To obtain the result for $n \geqslant 3$ we switch to the pressure formulation of the problem. In the proof of Lemma 2.1, the function $\psi_{0}$ has been defined by $p_{0}$. Here we want to define the pressure $p$ by the stream function $\psi$, which locally in $\Omega$ is a weak solution of

$$
\nabla \cdot\left(\frac{1}{r^{n-2}} \nabla \psi+\gamma e_{r}\right)=0,
$$


where the vector field under the divergence is in $L_{l o c}^{2}\left(\Omega ; \mathbb{R}^{2}\right)$. Since $\Omega$ is simply connected, there exists (up to an additive constant) a unique function $p \in H_{l o c}^{1,2}(\Omega)$ with

$$
\partial_{z} p=-\left(\frac{1}{r^{n-2}} \partial_{r} \psi+\gamma\right), \quad \partial_{r} p=\frac{1}{r^{n-2}} \partial_{z} \psi .
$$

Further, since $\partial_{r} \partial_{z} \psi=\partial_{z} \partial_{r} \psi$ in distributional sense, $p$ is a weak solution of

$$
\partial_{r}\left(r^{n-2} \partial_{r} p\right)+\partial_{z}\left(r^{n-2}\left(\partial_{z} p+\gamma\right)\right)=0 .
$$

Now consider the corresponding quantities on the $n$-dimensional domain $\tilde{\Omega}$, e.g.

$$
\tilde{p}\left(x_{1}, \ldots, x_{n}\right)=p\left(r, x_{n}\right) \quad \text { with } r=\sqrt{x_{1}^{2}+\cdots+x_{n-1}^{2}} .
$$

It follows that for $\tilde{\zeta} \in C_{0}^{\infty}(\tilde{\Omega})$, with $\tilde{\zeta}(x)=0$ for small $r$,

$$
\int_{\tilde{\Omega}} \nabla \tilde{\zeta} \cdot\left(\nabla \tilde{p}+\tilde{\gamma} e_{x_{n}}\right)=\int_{\Omega} r^{n-2} \nabla \zeta \cdot\left(\nabla p+\gamma e_{z}\right)=0,
$$

where

$$
\zeta(r, z)=\int \tilde{\zeta}(r \xi, z) d \mathcal{H}^{n-1}(\xi) .
$$

Moreover, since $\psi-\psi_{0} \in V$, it follows from (2.5) that $r^{2-n}|\nabla \psi|^{2} \in L^{1}\left(\Omega \backslash B_{\varepsilon}(W)\right)$. This implies that $r^{n-2}|\nabla p|^{2} \in L^{1}\left(\Omega \backslash B_{\varepsilon}(W)\right)$; that is, $|\nabla \tilde{p}| \in L^{2}\left(\tilde{\Omega} \backslash\{r=0\} \backslash B_{\varepsilon}(W)\right)$.

Since the axis $\{r=0\}$ is a removable singularity for $H^{1,2}$-spaces, it follows that $\tilde{p} \in$ $H_{l o c}^{1,2}(\tilde{\Omega} \backslash W)$ and $\int \tilde{\tilde{\Omega}} \cdot\left(\nabla \tilde{p}+\tilde{\gamma} e_{x_{n}}\right)=0$ for all $\tilde{\zeta} \in C_{0}^{\infty}(\tilde{\Omega} \backslash W)$. We then can apply the above technique to obtain

$$
\int_{B_{\rho}(x)}|\nabla \tilde{p}|^{2} \leqslant C \rho^{n-\varepsilon}
$$

locally in $\tilde{\Omega} \backslash W$. Covering $(n-2)$-dimensional rings by balls this gives

$$
\int_{\Omega \cap B_{\rho}\left(x_{0}\right)} r^{n-2}|\nabla p|^{2} \leqslant C\left(1+\left(\frac{r_{0}}{\rho}\right)^{n-2}\right) \rho^{n-\varepsilon}
$$

for balls $B_{\rho}\left(x_{0}\right)$ away from the well and $x_{0}=\left(r_{0}, z_{0}\right)$.

Since

$$
r^{2-n}|\nabla \psi|^{2} \leqslant 2 r^{n-2}\left(|\nabla p|^{2}+1\right),
$$

we obtain the estimate

$$
\int_{\Omega \cap B_{\rho}\left(x_{0}\right)} \frac{1}{r^{n-2}}|\nabla \psi|^{2} \leqslant C \rho^{2-\varepsilon} .
$$

Again, the Morrey lemma implies the Hölder continuity, at the axis and away from the well. 
THEOREM $3.6 \psi$ is Lipschitz continuous locally in $\Omega \cup\{(R, z) ; 0<z<1\}$.

Proof. We follow the proof of Lipschitz continuity in [3; see also 4: Theorem 3.8]. To include the boundary $\partial_{N} \Omega$ we reflect $\psi$ by

$$
\psi(r, z):=\psi(2 R-r, z) \text { for } R \leqslant r<2 R .
$$

Setting

$$
a(r, z):=\left\{\begin{array}{ll}
r^{2-n} & \text { for } r<R, \\
(2 R-r)^{2-n} & \text { for } r>R,
\end{array} \quad \beta(r, z):= \begin{cases}\gamma(r, z) & \text { for } r<R \\
-\gamma(2 R-r, z) & \text { for } r>R\end{cases}\right.
$$

we see that equation $(\star)$ in Section 2 becomes

$$
\int_{D} \nabla \zeta \cdot\left(a \nabla \psi+\beta e_{r}\right)=\int_{\partial_{N} \Omega} \zeta 2 \gamma_{N}
$$

for test functions $\zeta$ with support in $D:=\{(r, z) ; \quad 0<r<2 R, \quad 0<z<1\}$. Note that $a$ is locally Lipschitz continuous in $D$.

First we derive a monotonicity formula. Let

$$
\varphi(\rho):=\varphi_{1}(\rho) \cdot \varphi_{2}(\rho), \quad \varphi_{i}(\rho):=\int_{B_{\rho}} a\left|\nabla w_{i}\right|^{2},
$$

where $B_{\rho}=B_{\rho}\left(x_{0}\right)$ with $\psi\left(x_{0}\right)=Q_{s}$, and $w_{1}=\max \left(\psi-Q_{s}, 0\right), w_{2}=\min \left(\psi-Q_{s}, 0\right)$. We claim that

$$
\varphi^{\prime} \geqslant-2 L \varphi
$$

where $L$ is the local Lipschitz constant of $a$. To prove this replace $\zeta$ by $\zeta w_{i}$ and obtain

$$
\begin{aligned}
& \int_{D} \nabla\left(\zeta w_{i}\right) \cdot a \nabla w_{i}=2 \int_{\partial_{N} \Omega} \zeta w_{i} \gamma_{N}-\int_{D} \partial_{r}\left(\zeta w_{i}\right) \beta \\
& =c_{i}\left\{2 \int_{\partial_{N} \Omega} \zeta w_{i}-\int_{D \cap \Omega} \partial_{r}\left(\zeta w_{i}\right)+\int_{D \backslash \Omega} \partial_{r}\left(\zeta w_{i}\right)\right\}=0,
\end{aligned}
$$

where $c_{1}=0, c_{2}=1$ and $(\star \star)$ in Section 2 has been used. Letting $\xi \rightarrow \chi_{B_{\rho}}$ in an appropriate way, we derive that for almost all $\rho$

$$
\int_{B_{\rho}} a\left|\nabla w_{i}\right|^{2}=\int_{\partial B_{\rho}} a w_{i} \partial_{\rho} w_{i}
$$

Since

$$
\varphi^{\prime}(\rho)+\frac{4}{\rho} \varphi(\rho)=\sum_{i \neq j} \frac{\varphi_{j}(\rho)}{\rho^{2}} \int_{\partial B_{\rho}} a\left|\nabla w_{i}\right|^{2}
$$


we only have to consider the case $\varphi(\rho)>0$. Then

$$
\begin{aligned}
\rho \frac{\varphi^{\prime}(\rho)}{\varphi(\rho)}+4 & =\rho \sum_{i} \frac{\int_{\partial B_{\rho}} a\left(\left|\partial_{\rho} w_{i}\right|^{2}+\left|\frac{1}{\rho} \partial_{\theta} w_{i}\right|^{2}\right)}{\int_{\partial B_{\rho}} a w_{i} \partial_{\rho} w_{i}} \\
& \geqslant 2 \sum_{i} \frac{\left(\int_{\partial B_{\rho}} a\left|\partial_{\theta} w_{i}\right|^{2}\right)^{1 / 2}}{\left(\int_{\partial B_{\rho}} a\left|w_{i}\right|^{2}\right)^{1 / 2}} \geqslant 2 \sqrt{c_{\rho}} \sqrt{s_{i}},
\end{aligned}
$$

where

$$
c_{\rho}:=\frac{\inf _{B_{\rho}} a}{\sup _{B_{\rho}} a} \quad \text { and } \quad s_{i}:=\frac{\int_{\partial B_{\rho}}\left|\partial_{\theta} w_{i}\right|^{2}}{\int_{\partial B_{\rho}}\left|w_{i}\right|^{2}} .
$$

Since (see [3]) $\sqrt{s_{1}}+\sqrt{s_{2}} \geqslant 2$, we obtain

$$
\frac{\varphi^{\prime}(\rho)}{\varphi(\rho)} \geqslant-\frac{4}{\rho}\left(\sqrt{c_{\rho}}-1\right) .
$$

Since $c_{\rho} \geqslant 1-L \rho$, the assertion follows.

Next we derive a mean value estimate. Let again $B_{\rho}=B_{\rho}\left(x_{0}\right)$ with $\psi\left(x_{0}\right)=Q_{s}, G_{x}$ Green's function for the negative Laplacian with pole $x \in B_{\rho}$, and $P_{x}$ the corresponding Poisson function. Then, setting $u=\psi-Q_{s}$,

$$
u(x)-\int_{\partial B_{\rho}} P_{x} u=\int_{B_{\rho}} \nabla G_{x} \cdot \nabla \psi
$$

where the right-hand side is well defined by the $L^{2}$-gradient estimate in the previous proof. Using identity (3.1) with $\zeta=G_{x}$, it becomes

$$
=-\frac{1}{a(x)}\left\{\int_{B_{\rho}} \nabla G_{x} \cdot(a-a(x)) \nabla \psi+\int_{B_{\rho}} \nabla G_{x} \cdot \beta e_{r}+\int_{B_{\rho} \cap \partial_{N} \Omega} G_{x} 2 \gamma_{N}\right\} .
$$

Therefore we obtain for $x \in B_{\rho / 2}$

$$
\begin{aligned}
\left|u(x)-\int_{\partial B_{\rho}} P_{x} u\right| & \leqslant C\left\{\int_{B_{\rho}}|\nabla \psi|+\int_{B_{\rho}}\left|\nabla G_{x}\right|+\int_{B_{\rho} \cap \partial_{N} \Omega}\left|G_{x}\right|\right\} \\
& \leqslant C \rho\left(\|\nabla \psi\|_{L^{2}\left(B_{\rho}\right)}+1\right) \\
& \leqslant C \rho .
\end{aligned}
$$


For $x=x_{0}$ this gives

$$
\left|\int_{\partial B_{\rho}} u\right| \leqslant C \rho .
$$

Together with the monotonicity formula it follows as in [ACF2: Lemma 5.2] that

$$
\int_{\partial B_{\rho}}|u| \leqslant C \rho .
$$

Then the above estimate for $x \in B_{\rho / 2}$ implies

$$
\|u\|_{L^{\infty}\left(B_{\rho / 2}\right)} \leqslant C \rho .
$$

Finally, we use the fact that $\nabla \cdot a \nabla \psi=0$ in $\Omega \cap\left\{\psi \neq Q_{s}\right\}$ with smooth coefficient $a$. Let $x \in \Omega \cap\left\{\psi \neq Q_{s}\right\}$ near the free boundary, $\rho:=$ dist $\left(x,\left\{\psi=Q_{s}\right\}\right)$ and $x_{0} \in \partial B_{\rho}(x) \cap\left\{\psi=Q_{s}\right\}$. Then by the elliptic $C^{1, \alpha}$-estimate

$$
|\nabla \psi(x)| \leqslant C \frac{1}{\rho} f_{B_{\rho}(X)}\left|\psi-Q_{s}\right|,
$$

where the interior estimate was used if $B_{\rho / 2}(x) \subset \Omega$. Otherwise one has to apply the boundary estimate with homogeneous Neumann data. Since $B_{\rho}(x) \subset B_{\tilde{\rho} / 2}\left(x_{0}\right)$ with $\tilde{\rho}=4 \rho$, we obtain by the above $L^{\infty}$-estimate

$$
|\nabla \psi(x)| \leqslant C .
$$

\section{Free boundary}

The continuity and $z$-monotonicity of the solution $\psi$ in Section 3 imply that in $\Omega$, the boundary of $\left\{\psi>Q_{s}\right\}$ is the graph in the $z$-direction of an upper semicontinuous function, similarly the boundary of $\left\{\psi<Q_{s}\right\}$ is the graph of a lower semicontinuous function. We prove that the two functions coincide, i.e. a mushy region does not occur.

This essentially follows from the following

Non-oscillation Lemma 4.1 Suppose in $\Omega$ there are four vertical lines

$$
\ell_{i}:=\left\{\left(r_{i}, z\right) ; z_{1} \leqslant z \leqslant z_{2}\right\}, \quad i=1, \ldots, 4
$$

with $z_{1}<z_{2}, r_{1}<r_{2}<r_{3}<r_{4}$, such that $\psi-Q_{s}$ has no zeros on these lines and changes sign on successive lines, for instance as in Fig. 9.

Then

$$
z_{2}-z_{1} \leqslant \frac{2 L}{r_{1}^{n-2}}\left(r_{4}-r_{1}\right),
$$

where $L$ is the Lipschitz constant of $\psi$ on the rectangle enclosing the four lines. 


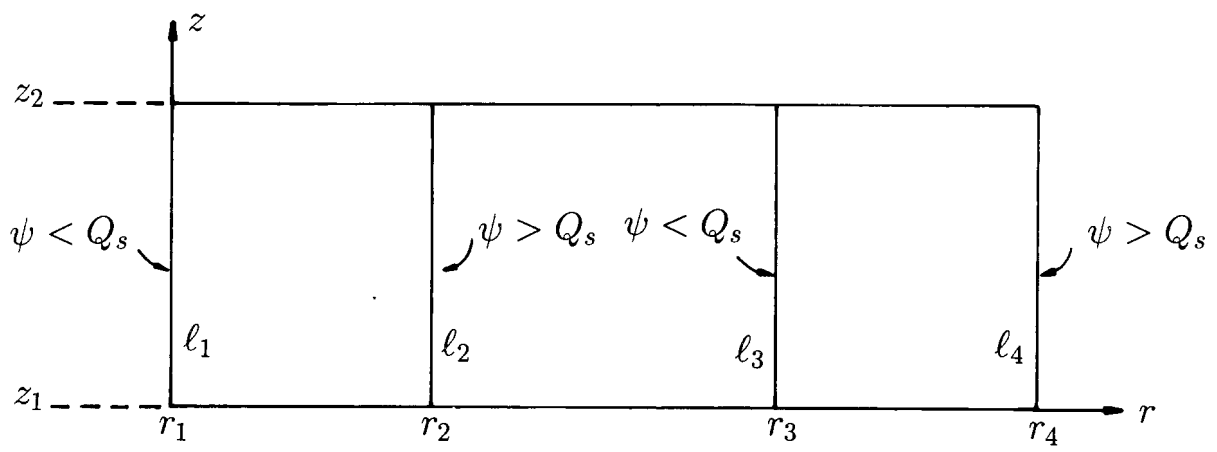

FIG. 9. Sign change of $\psi-Q_{s}$ on successive lines.

Proof. We use the following. In the open set $\left\{\psi \neq Q_{s}\right\}$, the stream function solves the analytic elliptic equation

$$
\partial_{r}\left(\frac{1}{r^{n-2}} \partial_{r} \psi\right)+\partial_{z}\left(\frac{1}{r^{n-2}} \partial_{z} \psi\right)=0
$$

It follows that at each point $(r, z) \in \Omega$ with $\psi(r, z) \neq Q_{s}$, either $\nabla \psi(r, z) \neq 0$ or that $\nabla \psi(r, z)=0$ and the level set $\{\psi=\psi(r, z)\}$ near $(r, z)$ consists of an even number of smooth lines:

either

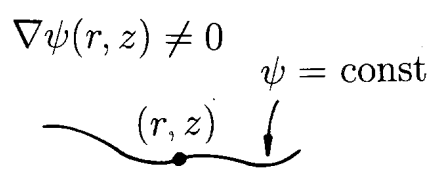

or

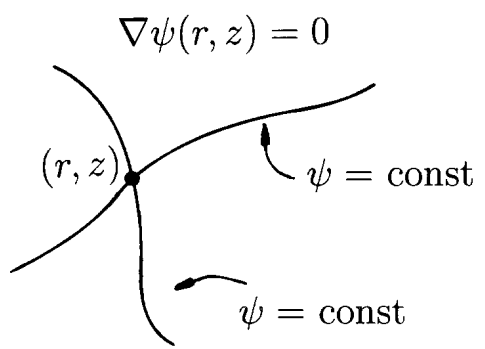

In particular, points with the latter property are isolated. By an arbitrary small perturbation of $z_{1}, z_{2}$ we can assume that

$$
\nabla \psi(r, z) \neq 0 \text { for } r_{1} \leqslant r \leqslant r_{4}, z=z_{1} \text { or } z_{2} \text {. }
$$

For definiteness, let us assume that $\psi<Q_{s}$ on $\ell_{1}$. Consider the rectangle

$$
R_{1}:=\left[r_{1}, r_{3}\right] \times\left[z_{1}, z_{2}\right] .
$$

Choose $\xi_{1}$ with $r_{1}<\xi_{1}<r_{3}$ so that

$$
\psi\left(\xi_{1}, z_{1}\right)=\sup _{r_{1} \leqslant r \leqslant r_{3}} \psi\left(r, z_{1}\right)>Q_{s} .
$$


Since $\partial_{z} \psi \geqslant 0$ by Lemma 3.4 and $\nabla \psi\left(\xi_{1}, z_{1}\right) \neq 0$ by the choice of $z_{1}$, it follows that $\nabla \psi\left(\xi_{1}, z_{1}\right)$ points upwards, i.e. $\partial_{z} \psi\left(\xi_{1}, z_{1}\right)>0$. We thus can construct in $R_{1}$ a curve $s \mapsto \sigma_{1}(s)$, with $\sigma_{1}(0)=$ $\left(\xi_{1}, z_{1}\right)$, so that

$$
\sigma_{1}^{\prime}(s)=\frac{\nabla \psi\left(\sigma_{1}(s)\right)}{\left|\nabla \psi\left(\sigma_{1}(s)\right)\right|} \quad \text { whenever } \nabla \psi\left(\sigma_{1}(s)\right) \neq 0,
$$

and so that $\sigma_{1}$ is Lipschitz continuous. By the properties of $\psi$ on $\partial R_{1}$ it follows that the curve reaches a point $\left(\tilde{\xi}_{1}, z_{2}\right)=\sigma_{1}\left(s_{1}\right)$ on $\partial R_{1}$, as in Fig. 10.

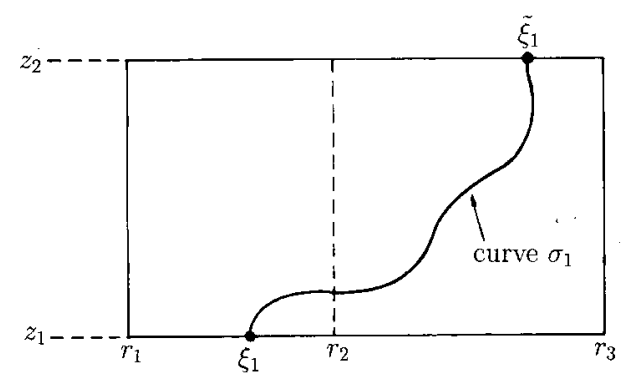

FIG. 10. Construction of the curve $\sigma_{1}$ in $R_{1}$.

Similarly, let

$$
R_{2}:=\left[r_{2}, r_{4}\right] \times\left[z_{1}, z_{2}\right]
$$

and choose $\xi_{2}$, with $\max \left(r_{2}, \tilde{\xi}_{1}\right)<\xi_{2}<r_{4}$, so that

$$
\psi\left(\xi_{2}, z_{2}\right)=\inf _{\max \left(r_{2}, \tilde{\xi}_{1}\right) \leqslant r \leqslant r_{4}} \psi\left(r, z_{2}\right)<Q_{s} .
$$

As above, $\nabla \psi\left(\xi_{2}, z_{2}\right)$ points upwards, so that there exists a curve $s \mapsto \sigma_{2}(s)$ in $R_{2}$, with $\sigma_{2}(0)=$ $\left(\xi_{2}, z_{2}\right)$, so that

$$
\sigma_{2}^{\prime}(s)=-\frac{\nabla \psi\left(\sigma_{2}(s)\right)}{\left|\nabla \psi\left(\sigma_{2}(2)\right)\right|} \quad \text { whenever } \nabla \psi\left(\sigma_{2}(s)\right) \neq 0 .
$$

As before, this curve reaches a point $\left(\tilde{\xi}_{2}, z_{1}\right)=\sigma_{2}\left(s_{2}\right)$ on $\partial R_{2}$.

Since $\psi>Q_{s}$ on $\sigma_{1}$ and $\psi<Q_{s}$ on $\sigma_{2}$ it follows that $\tilde{\xi}_{2}>\xi_{1}$. Let $R$ be the region bounded by $\sigma_{1}, \sigma_{2}$ and the horizontal segments $\left\{\left(r, z_{1}\right): \xi_{1} \leqslant r \leqslant \tilde{\xi}_{2}\right\}$ and $\left\{\left(r, z_{2}\right): \tilde{\xi}_{1} \leqslant r \leqslant \xi_{2}\right\}$, see Fig. 11

The idea is now to integrate $\Delta \psi$ over $R$ and to use on the one hand Gauss' theorem and on the other hand the differential equation. To make this precise, we have to use the weak equation for $\psi$ with test function

$$
\zeta(r, z)=\eta(z) d_{\rho}(r, z), \quad \rho>0 \text { small },
$$

where

$$
d_{\rho}(r, z)=\min \left(1, \frac{1}{\rho} \operatorname{dist}((r, z), \partial R)\right),
$$




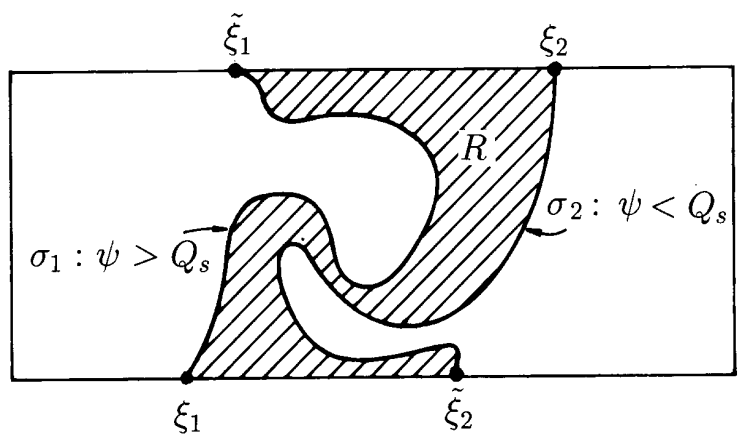

FIG. 11. Construction of the region $R$.

and where $\eta$ is a cut-off function $\eta \in C_{0}^{\infty}(] z_{1}, z_{2}[)$ with $0 \leqslant \eta \leqslant 1$. Then

$$
\begin{aligned}
\int_{R} \frac{1}{r^{n-2}} \nabla \zeta \cdot \nabla \psi & =-\int_{R} \nabla \zeta \cdot\left(\gamma e_{r}\right) \\
& =-\int_{R} \eta \gamma \partial_{r} d_{\rho} .
\end{aligned}
$$

For small $\rho$ we have $\partial_{r} d_{\rho} \neq 0$ only near $\sigma_{2}$, where $\gamma=1$, and near $\sigma_{1}$, where $\gamma=0$. Hence the right-hand side integral becomes

$$
\begin{gathered}
-\int_{z_{1}}^{z_{2}} \eta(z)\left(\int_{\left\{r ;(r, z) \in R \cap B_{\rho}\left(\sigma_{2}\right)\right\}} \partial_{r} d_{\rho}(r, z) \mathrm{d} r\right) \mathrm{d} z \\
=\int_{z_{1}}^{z_{2}} \eta(z) \mathrm{d} z .
\end{gathered}
$$

Thus

$$
\int_{z_{1}}^{z_{2}} \eta(z) \mathrm{d} z=\int_{R} \frac{1}{r^{n-2}} d_{\rho} \partial_{z} \eta \partial_{z} \psi+\int_{R} \frac{\eta}{r^{n-2}} \nabla d_{\rho} \cdot \nabla \psi .
$$

The last integral tends to 0 as $\rho \rightarrow 0$, since $\nabla \psi$ is tangential on $\sigma_{1}, \sigma_{2}$ by construction of these 
curves. Hence we obtain

$$
\begin{aligned}
\int_{z_{1}}^{z_{2}} \eta(z) \mathrm{d} z & \leqslant \int_{R} \frac{1}{r^{n-2}}\left|\partial_{z} \eta\right|\left|\partial_{z} \psi\right| \\
& \leqslant \frac{L}{r_{1}^{n-2}} \int_{R}\left|\partial_{z} \eta\right| \leqslant \frac{L\left(r_{4}-r_{1}\right)}{r_{1}^{n-2}} \int_{z_{1}}^{z_{2}}\left|\partial_{z} \eta(z)\right| \mathrm{d} z
\end{aligned}
$$

Now choosing $\eta$ as in Fig. 12 and letting $\varepsilon \searrow 0$ we obtain the assertion.

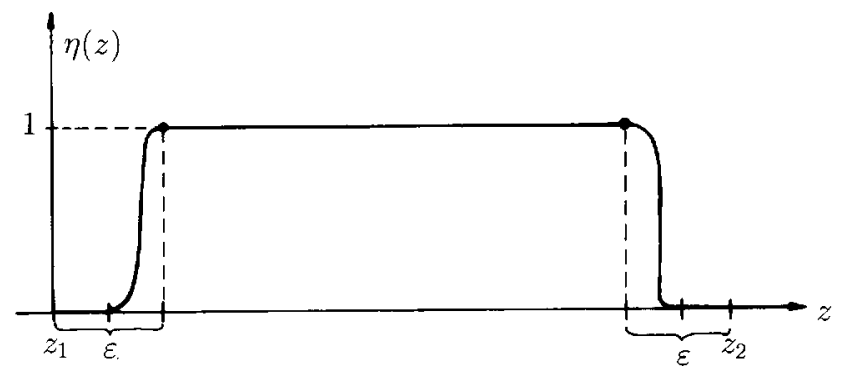

FIG. 12. Properties of the test function $\eta$.

Next we show

PROPOSITION 4.2 A free boundary cannot contain isolated vertical segments: i.e. a situation as below cannot occur.

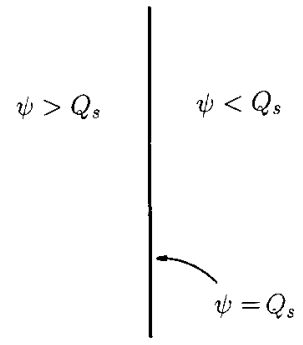

FIG. 13. Isolated vertical segment.

Proof. See also [4]. It follows that $\partial_{z} \gamma=0$ in distributional sense in a neighbourhood $U$ of the segment. For small $\delta>0$, consider the function $v(r, z):=\psi(r, z+\delta)-\psi(r, z)$. Then $v \geqslant 0$ by 
Proposition 3.4, $v=0$ on the segment, and $v$ is a continuous solution of $\nabla \cdot\left(r^{2-n} \nabla v\right)=0$ in $U$. By elliptic regularity theory $v$ is smooth, and therefore $v=0$ by the strong maximum principle. We conclude that $\psi(r, z)=\varphi(r)$ in $U$ with a continuous function $\varphi$ different from $Q_{s}$ away from the segment. This argument extends to small vertical strips, on the left of the segment reaching the top of $\Omega$ and on the right of the segment reaching the bottom of $\Omega$. This yields a contradiction to the Dirichlet data.

Next we show that a mushy region (if it exists) increases to the left.

LEMMA 4.3 Suppose there exist $\left.r_{0} \in\right] 0, R\left[\right.$ and $\left.z_{1}, z_{2} \in\right] 0,1\left[\right.$, with $z_{1}<z_{2}$, such that

$$
\psi\left(r_{0}, z\right) \begin{cases}>Q_{s} & \text { for } z_{2}<z<1 \\ =Q_{s} & \text { for } z_{1} \leqslant z \leqslant z_{2} \\ <Q_{s} & \text { for } 0<z<z_{1} .\end{cases}
$$

Then

$$
\left.\left.\psi=Q_{s} \text { in }\right] 0, r_{0}\right] \times\left[z_{1}, z_{2}\right]
$$

Proof. Consider two points $T=\left(r_{0}, z_{T}\right)$ and $B=\left(r_{0}, z_{B}\right)$ with $z_{1} \leqslant z_{B}<z_{T} \leqslant z_{2}$, and two sequences $\left(x_{n}\right)_{n \in \mathbb{N}}, \quad\left(\tilde{x}_{n}\right)_{n \in \mathbb{N}}$ with $x_{n}=\left(r_{n}, z_{n}\right), \quad r_{n}<r_{0}, x_{n} \rightarrow T$ as $n \rightarrow \infty$ and $\tilde{x}_{n}=$ $\left(\tilde{r}_{n}, \tilde{z}_{n}\right), \tilde{r}_{n}<r_{0}, \tilde{x}_{n} \rightarrow B$ as $n \rightarrow \infty$, see Fig. 14. Now assume that $\psi\left(x_{n}\right)<Q_{s}$ and $\psi\left(\tilde{x}_{n}\right)>Q_{s}$ for large $n$.

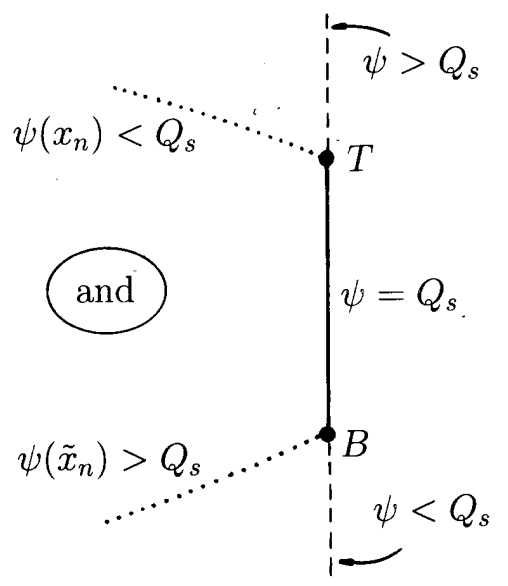

FIG. 14. Sequences $\left(x_{n}\right)_{n \in \mathbb{N}}$ and $\left(\tilde{x}_{n}\right)_{n \in \mathbb{N}}$.

Then in view of the non-oscillation result, these sequences cannot exist simultaneously. Therefore we have to deal with one of the following two cases.

Case 1. $\psi \geqslant Q_{s}$ in a left neighbourhood $N$ of $T$. 


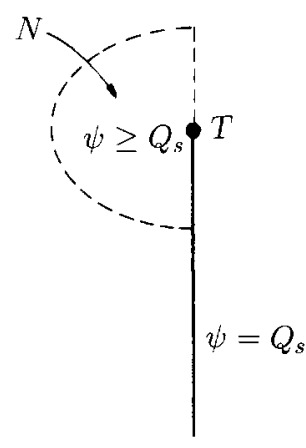

top case

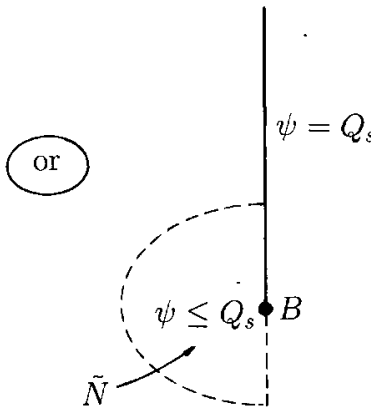

bottom case

FIG. 15. Two possible cases.

Case 2. $\psi \leqslant Q_{s}$ in a left neighbourhood $\tilde{N}$ of $B$.

In the first case we assert

CLAIM $4.4 \psi=Q_{s}$ in $\left.\left.L:=N \cap\right] 0, r_{0}\right] \times\left[z_{B}, z_{T}\right]$.

Proof. Suppose the assertion is not true. Then there exists a point $x \in L$, where $\psi(x)>Q_{s}$. The Hölder continuity of $\psi$ implies the existence of a neighbourhood $M$ where $\psi>Q_{s}$ and consequently $\gamma=0$, see Fig. 16.

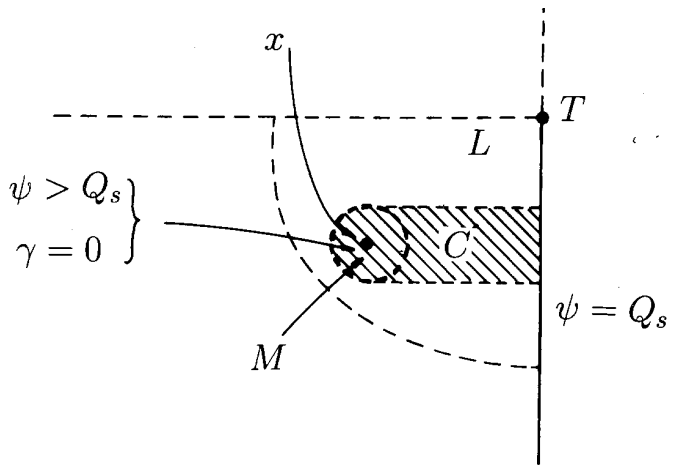

FIG. 16. Construction of sets.

Next, consider the set $C \subset L$ shown as the shaded region in Fig. 16. Since $\psi \geqslant Q_{s}$ in $C$ we 
have

$$
\nabla \cdot\left(r^{2-n} \nabla \psi\right) \geqslant 0 \text { and thus } \partial_{r} \gamma \leqslant 0 \text { in } C,
$$

by the differential equation for $\psi$. Using $\gamma \geqslant 0$ in $\Omega$ and $\gamma=0$ in $M$, we find $\gamma=0, \nabla$. $\left(r^{2-n} \nabla \psi\right)=0$ and, by the strong maximum principle, $\psi>Q_{s}$ in $C$.

Next we choose points $P \neq \tilde{P}$ as in Fig. 17. By the non-oscillation result (argue as for cases 1,2 above, but now from the right instead of the left) we have either $\psi \geqslant Q_{s}$ in a small horizontal strip to the right of the point $P$, or $\psi \leqslant Q_{s}$ in a small horizontal strip to the right of $\tilde{P}$.

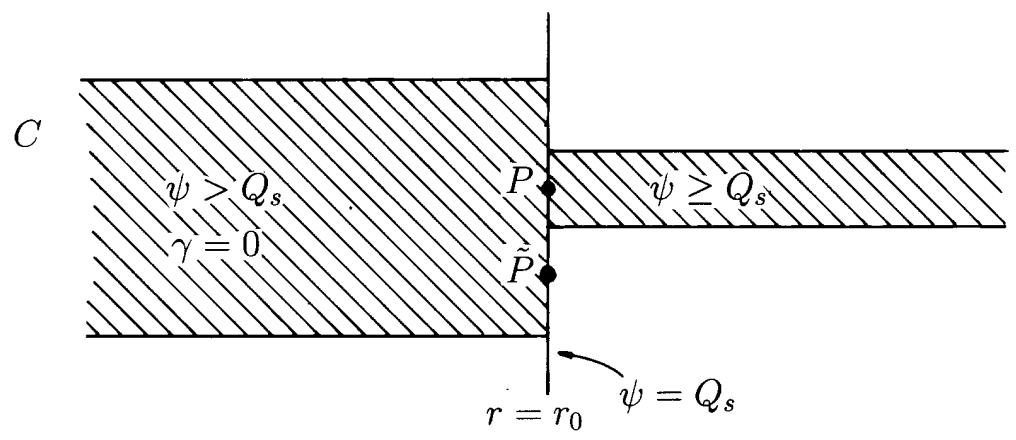

FIG. 17. Possible situation near $P$.

First consider $\psi \geqslant Q_{s}$ in a right neighbourhood of $P$. This implies again $\nabla \cdot\left(r^{2-n} \nabla \psi\right) \geqslant 0$ and $\partial_{r} \gamma \leqslant 0$ in the shaded area of Fig. 17. Since $\gamma=0$ in $C$, we have $\gamma=0, \nabla \cdot\left(r^{2-n} \nabla \psi\right)=0$ and thus $\psi>Q_{s}$ in the shaded area. In particular, $\psi(P)>Q_{s}$ which gives a contradiction.

Finally, consider the case $\psi \leqslant Q_{s}, \psi \neq \equiv Q_{s}$, in every small right neighbourhood of $\tilde{P}$. If $x^{\prime}=\left(r^{\prime}, z^{\prime}\right)$ is a point in such a neighbourhood with $\psi\left(x^{\prime}\right)<Q_{s}$ then, using similar arguments as before, $\psi<Q_{s}$ in the shaded region of Fig. 18. In particular, $\psi\left(r^{\prime}, z^{\prime}\right)<Q_{s}$ for $r>r^{\prime}$. Since $\partial_{z} \psi \geqslant 0$, this implies $\psi<Q_{s}$ and $\gamma=1$ in the region with upper left corner $x^{\prime}$, see again Fig. 18 .
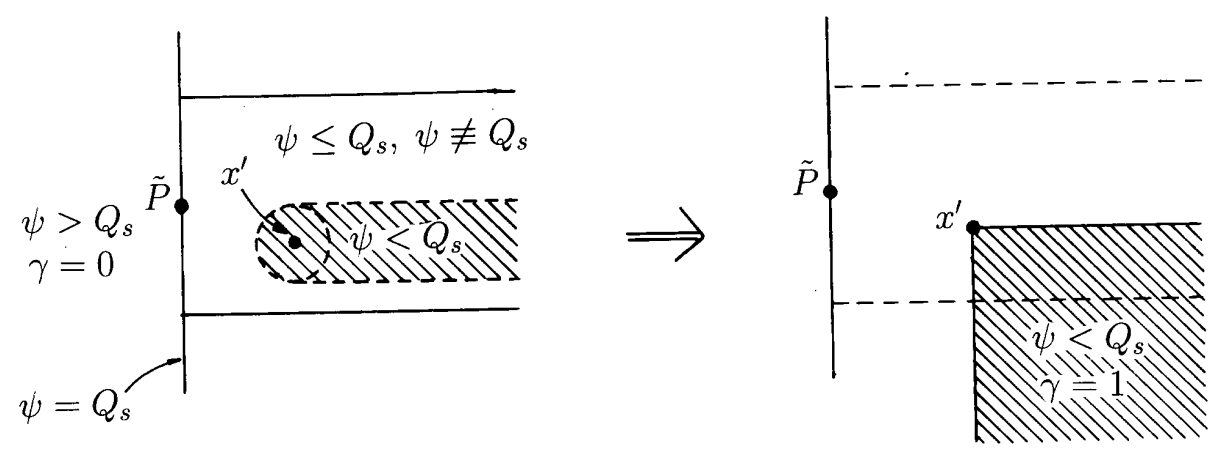

FIG. 18. Construction in a right neighbourhood. 
By assumption, there exists a sequence $\left(x_{n}^{\prime}=\left(r_{n}^{\prime}, z_{n}^{\prime}\right)\right)_{n \in \mathbb{N}}$ with $x_{n}^{\prime} \rightarrow \tilde{P}$ as $n \rightarrow \infty, r_{n}>r_{0}$ and $\psi\left(x_{n}^{\prime}\right)<Q_{s}$. As a consequence of Fig. 18, we therefore arrive at the following situation which is a contradiction to Proposition 4.2. This concludes the proof of the claim.

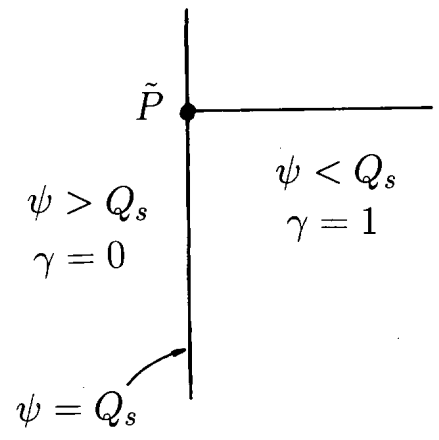

We continue with the proof of Lemma 4.3 using Claim 4.4. Assuming the top case of Fig. 15, we find $\psi=Q_{s}$ in $L$ and, using $\partial_{z} \psi \geqslant 0, \psi \leqslant Q_{s}$ below $L$. Thus the top case implies the bottom case. Conversely, starting from the bottom case of Fig. 15, we find $\psi=Q_{s}$ in an upper left neighbourhood of $B$ (repeat proof of Claim 4.4 with reversed signs). Using again the $z$-monotonicity of $\psi$ we observe that the bottom case implies the top case. Therefore both cases are always true. Thus, by Claim 4.4, we are left with $\psi=Q_{s}$ in $L$, and similarly $\psi=Q_{s}$ in some left upper neighbourhood of $B$. This gives $\psi=Q_{s}$ to the left of the segment $B T$, which proves the lemma.

With these preparations we can prove

THEOREM 4.5 The free boundary $\Omega \cap\left\{\psi=Q_{s}\right\}$ is the graph in the $z$-direction of a continuous function $g:] 0, R[\rightarrow \mathbb{R}$. Moreover,

$$
\left.h=\lim _{r \searrow 0} g(r) \quad \text { and } \quad u_{s}:=\lim _{r \nearrow R} g(r) \in\right] 0,1[
$$

exists.

Proof. From the continuity of $\psi$ in Lemma 3.5 it follows that the free boundary stays away from the lower and upper boundary of $\Omega$. Therefore, if the free boundary is not a continuous graph, a vertical segment as in Lemma 4.3 exists. However, then we conclude that $\psi=Q_{s}$ to the left, and therefore $\psi$ cannot attain its boundary values on $\{r=0\}$, which are either 0 or $Q$. Hence $g$ exists and by the continuity of Theorem 3.5 we infer that $g(r) \rightarrow h$ as $r \searrow 0$. The last statement follows from the Non-Oscillation Lemma 4.1, taking into account that $\psi$ is Lipschitz continuous up to the right boundary of $\Omega$ (Theorem 3.6.).

REMARK 4.6 Theorem 4.5 implies that $\gamma=\chi_{\{z<g(r)\}}$ and $\gamma_{N}=\chi_{\left\{z<u_{s}\right\}}$. Moreover, we find for fixed $z \in] 0, u_{s}[\cup] u_{s}, 1[$

$$
\lim _{r \nearrow R} \gamma(r, z)=\gamma_{N}(z) .
$$

As a consequence, $\psi$ satisfies the Neumann condition on $\partial_{N} \Omega$, away from the free boundary point. 


\section{Free boundary near well}

It follows from Theorem 4.5 that the free boundary approaches $W$ as a continuous curve. In this section we prove that the free boundary has a tangent at $W$ and that it approaches $W$ in a $C^{1}$-sense.

We use polar coordinates.

$$
r+i(z-h)=\rho e^{i \theta}
$$

and consider a small neighbourhood

$$
D_{\rho_{0}}:=\left\{(r, z) \in \Omega ; r^{2}+(z-h)^{2}<\rho_{0}^{2}\right\} .
$$

The function $\psi_{*}$ defined in Lemma 2.1 plays an important role in this section as well as in Section 6. It has the form

$$
\psi_{*}(r, z)=\frac{Q}{2}\left(\varphi_{*}(r, z)+1\right)
$$

with

$$
\varphi_{*}(r, z)=\tilde{\varphi}_{*}(\theta)= \begin{cases}\frac{2}{\pi} \theta & \text { for } n=2, \\ \sin \theta & \text { for } n=3,\end{cases}
$$

implying that $\psi_{*}$ is constant on rays starting at $W$. We therefore set

$$
\tilde{\psi}_{*}(\theta)=\frac{Q}{2}\left(\tilde{\varphi}_{*}(\theta)+1\right) .
$$

As $\psi_{0}$, the function $\psi_{*}$ satisfies

$$
\partial_{r}\left(r^{2-n} \partial_{r} \psi_{*}\right)+\partial_{z}\left(r^{2-n} \partial_{z} \psi_{*}\right)=0 .
$$

Hence, the local weak equation of $\psi$ near $W$ can be written as

$$
\int_{D_{\rho_{0}}} \nabla \zeta \cdot\left(r^{2-n} \nabla\left(\psi-\psi_{*}\right)+\gamma e_{r}\right)=0
$$

for all $\zeta \in C_{0}^{\infty}\left(D_{\rho_{0}}\right)$.

Definition 5.1 Define $\omega_{*}$, with $-\frac{\pi}{2}<\omega_{*}<\frac{\pi}{2}$, by

$$
\tilde{\psi}_{*}\left(\omega_{*}\right)=Q_{s} .
$$

Thus $\psi_{*}=Q_{s}$ on the ray in the direction $e^{i \omega_{*}}$. We have

$$
\tilde{\varphi}_{*}\left(\omega_{*}\right)=Q_{r e l}:=\frac{Q_{s}-Q_{f}}{Q_{s}+Q_{f}},
$$

that is

$$
\omega_{*}= \begin{cases}\frac{\pi}{2} Q_{\text {rel }} & \text { for } n=2, \\ \arcsin Q_{\text {rel }} & \text { for } n=3 .\end{cases}
$$


We shall need the following.

Comparison Lemma 5.2 Let $D \subset \Omega$ be open and connected, and let $\psi \in C_{l o c}^{0,1}(D), \gamma=$ $1-H\left(\psi-Q_{s}\right)$ be a weak solution of

$$
\nabla \cdot\left(r^{2-n} \nabla \psi+\gamma e_{r}\right)=0 \text { in } D .
$$

Further, let $\varphi \in C_{l o c}^{0,1}(D), \beta=1-H\left(\varphi-Q_{s}\right)$ be a smooth strict supersolution in the sense that

$$
\begin{gathered}
\nabla \cdot\left(r^{2-n} \nabla \varphi\right)=0 \text { in } D \cap\left\{\varphi \neq Q_{s}\right\}, \\
D \cap\left\{\varphi=Q_{s}\right\} \text { is a } C^{2} \text {-curve, } \\
{\left[v \cdot\left(r^{2-n} \nabla \varphi+\beta e_{r}\right)\right]<0 \text { on } D \cap \partial\left\{\varphi<Q_{s}\right\} .}
\end{gathered}
$$

Then $\psi \leqslant \varphi$ in $D$ implies $\psi<\varphi$ in $D$.

Note. The corresponding version for strict subsolutions also holds.

Proof. Let $x_{0}=\left(r_{0}, z_{0}\right) \in D$ with $\psi\left(x_{0}\right)=\varphi\left(x_{0}\right)$. If $\psi\left(x_{0}\right)>Q_{s}$, then the strong maximum principle implies that $\psi=\varphi$ in the connected component of $\left\{\psi>Q_{s}\right\}$ containing $x_{0}$. The same argument applies if $\psi\left(x_{0}\right)<Q_{s}$. Thus it remains to exclude the case that $\psi\left(x_{0}\right)=Q_{s}$. We consider the blow-up at $x_{0}$. Since $\varphi$ has a $C^{2}$ free boundary, the functions

$$
\varphi_{\delta}(x):=\frac{1}{\delta}\left(\varphi\left(x_{0}+\delta x\right)-\varphi\left(x_{0}\right)\right)
$$

converge to a piecewise linear function $\varphi_{0}$, and $\left\{\varphi_{0}=0\right\}$ is a line through the origin. Moreover,

$$
\left[v \cdot\left(r_{0}^{2-n} \nabla \varphi_{0}+\beta_{0} e_{r}\right)\right]<0
$$

on this line, where $\beta_{0}=1-H\left(\varphi_{0}\right)$. Similarly

$$
\begin{aligned}
& \psi_{\delta} \rightarrow \psi_{0} \text { weakly in } H_{l o c}^{1,2}\left(\mathbb{R}^{2}\right), \\
& \gamma_{\delta} \rightarrow \gamma_{0} \text { weak star in } L_{l o c}^{\infty}\left(\mathbb{R}^{2}\right),
\end{aligned}
$$

where $\psi_{0}$ is globally Lipschitz continuous with $\psi_{0}(0)=0$ and

$$
\nabla \cdot\left(r_{0}^{2-n} \nabla \psi_{0}+\gamma_{0} e_{r}\right)=0 \quad \text { in } \quad \mathbb{R}^{2} .
$$

Furthermore, as in [4: Lemma 3.10],

$$
\nabla \psi_{\delta} \rightarrow \nabla \psi_{0} \text { strongly in } L_{l o c}^{2}\left(\mathbb{R}^{2}\right) .
$$

Following again [4: Lemma 3.10] and using the monotonicity formula derived in the proof of Theorem 3.6, we arrive at the following two cases:

(i) $\psi_{0}$ is a piecewise linear two-phase solution. Then $\left\{\psi_{0}=0\right\}=\left\{\varphi_{0}=0\right\}$. Since $\psi_{0} \leqslant$ $\varphi_{0}, \psi_{0}$ satisfies the free boundary condition, and $\varphi_{0}$ the strict free boundary condition for a supersolution, we end up with a contradiction. 
(ii) $\psi_{0}$ has a sign. Since $\psi_{0} \leqslant \varphi_{0}$ and $\left\{\varphi_{0}<0\right\}$ is non-empty, $\psi_{0} \leqslant 0$ is a one-phase solution. Further, $\min \left(\varphi_{0}, 0\right)$ is a strict one-phase supersolution at its free boundary. Then we apply the bump argument [see 4: proof of Lemma 5.2] to derive a contradiction.

The next statement implies, that the free boundary has the unique tangent direction $e^{i \omega_{*}}$ at the well.

LEMMA 5.3 For small $\rho>0$ there exist $\varepsilon_{\rho}>0$ with $\varepsilon_{\rho} \rightarrow 0$ as $\rho \rightarrow 0$ so that

$$
\psi_{*}-\varepsilon_{\rho} \leqslant \psi \leqslant \psi_{*}+\varepsilon_{\rho} \quad \text { in } \quad D_{\rho} .
$$

Proof. Let $\varepsilon \neq 0$ be small, $-\frac{\pi}{2}<\omega_{\varepsilon}<\frac{\pi}{2}$, and define

$$
\tilde{\varphi}(\theta):= \begin{cases}\varepsilon+\frac{\tilde{\psi}_{*}(\theta)}{\tilde{\psi}_{*}\left(\omega_{\varepsilon}\right)}\left(Q_{s}-\varepsilon\right) & \text { for }-\frac{\pi}{2} \leqslant \theta \leqslant \omega_{\varepsilon}, \\ Q+\varepsilon-\frac{Q-\tilde{\psi}_{*}(\theta)}{Q-\tilde{\psi}_{*}\left(\omega_{\varepsilon}\right)}\left(Q+\varepsilon-Q_{s}\right) & \text { for } \omega_{\varepsilon} \leqslant \theta \leqslant \frac{\pi}{2} .\end{cases}
$$

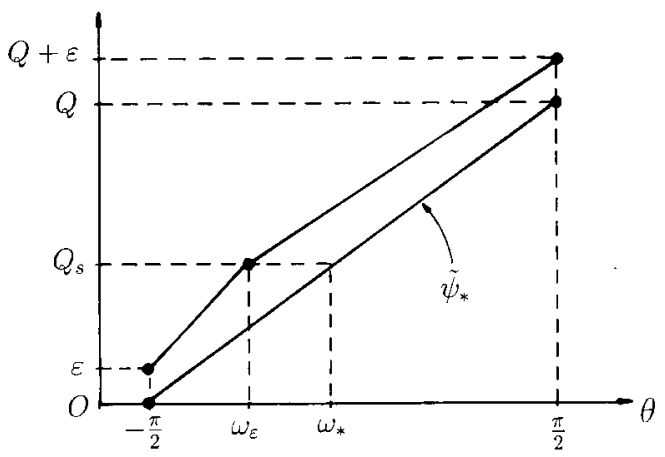

FIG. 19. $\tilde{\psi}$ for $n=2$ and $\varepsilon>0$.

Then

$$
\tilde{\varphi}^{\prime}\left(\omega_{\varepsilon}-0\right)(\gtrless) \tilde{\varphi}^{\prime}\left(\omega_{\varepsilon}+0\right)
$$

if and only if

$$
\tilde{\psi}_{*}\left(\omega_{\varepsilon}\right)(\lessgtr) \tilde{\psi}_{*}\left(\omega_{*}\right)-\varepsilon .
$$

For small $|\varepsilon|>0$ this is satisfied if $\omega_{\varepsilon}=\omega_{*}-a \varepsilon$, with $a>1 / \tilde{\psi}_{*}^{\prime}\left(\omega_{*}\right)>0$. We define

$$
\varphi(r, z):=\tilde{\varphi}(\theta) \text { with } \theta \text { as in (5.1). }
$$


Then on the free boundary of $\varphi$

$$
\left[v \cdot r^{2-n} \nabla \varphi\right](r, z)=r^{1-n}\left[\tilde{\varphi}^{\prime}\right](\theta) \rightarrow-\infty(+\infty),
$$

as $(r, z) \in \partial\left\{\varphi<Q_{s}\right\} \rightarrow W$. It follows that $\varphi$ is a smooth strict super-(sub-) solution in $D_{\rho_{\varepsilon}}$ in the sense of Lemma 5.2, if $\rho_{\varepsilon}$ is small enough. Moreover, if $|\varepsilon|$ is small enough, then $\partial_{z} \varphi>0$.

In order to compare $\psi$ with $\varphi$ we use the fact that $\nabla\left(\psi-\psi_{*}\right) \in L^{2}\left(D_{\rho_{0}}\right)$. Then by the Courant Lemma [C], there is a countable sequence $\rho \rightarrow 0$ with

$$
\underset{\left[-\frac{\pi}{2}, \frac{\pi}{2}\right]}{\operatorname{Osc}}\left(\tilde{\psi}-\tilde{\psi}_{*}\right)(\rho, \cdot) \rightarrow 0
$$

Since $\tilde{\psi}\left(\rho,-\frac{\pi}{2}\right)=0=\tilde{\psi}_{*}\left(-\frac{\pi}{2}\right)$, we can choose $\rho<\rho_{\varepsilon}$ so that

$$
\left|\psi-\psi_{*}\right|<|\varepsilon| \text { on } \Omega \cap \partial D_{\rho} .
$$

Then on this set $\psi(\lessgtr) \psi_{*}+\varepsilon(\lesseqgtr) \varphi$ and therefore

$$
\psi(\lessgtr) \varphi \quad \text { on } \quad \partial D_{\rho} \backslash W .
$$

To apply Lemma 5.2, let $\varphi_{\delta}(r, z):=\varphi(r, z+\delta)$ for $\delta>0(<0)$. Obviously $\psi(\lesseqgtr) \varphi_{\delta}$ in $\bar{D}_{\rho}$ for large $|\delta|$. Here we take the value $Q$ for $\psi$ at $W$ and $\varepsilon$ for $\varphi_{\delta}$ at $W-\delta e_{z}\left(0\right.$ for $\psi$ and $Q+\varepsilon$ for $\left.\varphi_{\delta}\right)$. Choose $|\delta|$ minimal with this property. Assume $|\delta|>0$. Then $\psi(\lessgtr) \varphi_{\delta}$ in $D_{\rho}$ by Lemma 5.2. Since $\partial_{z} \varphi>0$ we still have $\psi(\lessgtr) \varphi_{\delta}$ on $\partial D_{\rho}$, which contradicts the minimality of $|\delta|$. Thus $|\delta|=0$ and consequently

$$
\psi(\lesseqgtr) \varphi(\lesseqgtr) \psi_{*}+C \varepsilon \text { in } D_{\rho} .
$$

For later use we introduce the scaling

$$
\psi_{\rho}(x):=\psi(W+\rho(x-W)) \text { for } x=(r, z) \text { near } W,
$$

the same for $\gamma_{\rho}$. Equation (5.2) then becomes

$$
\int_{D_{\rho_{0} / \rho}} \nabla \zeta \cdot\left(r^{2-n} \nabla\left(\psi_{\rho}-\psi_{*}\right)+\rho^{n-1} \gamma_{\rho} e_{r}\right)=0
$$

for $\zeta \in C_{0}^{\infty}\left(D_{\rho_{0} / \rho}\right)$. Let $R>0$ be a fixed large number and $\rho \ll \rho_{0} / R$.

From Lemma 5.3 we infer

$$
\begin{gathered}
\left|\psi_{\rho}-\psi_{*}\right| \leqslant \varepsilon_{\rho} \rightarrow 0 \quad \text { as } \rho \rightarrow 0 \text { in } D_{R}, \\
\left\{\psi_{\rho}=Q_{s}\right\} \cap D_{R} \subset\left\{W+s e^{i \theta} ; \quad s>0,\left|\theta-\omega_{*}\right| \leqslant \frac{2 \varepsilon_{\rho}}{Q}\right\} .
\end{gathered}
$$

PROPOSITION 5.4 For $\sigma>0$ consider the region

$$
G_{R}^{\sigma}:=\left\{(r, z)=W+s e^{i \theta} \in \bar{D}_{R} ; s \geqslant \sigma,\left|\theta-\omega_{*}\right| \geqslant \sigma\right\} .
$$

Then for small $\rho$

$$
\left|\nabla\left(\psi_{\rho}-\psi_{*}\right)\right| \leqslant C(\sigma, R) \varepsilon_{\rho} \quad \text { in } \quad G_{R}^{\sigma}
$$


Proof. For small $\rho$ we have $\nabla \cdot\left(r^{2-n} \nabla\left(\psi_{\rho}-\psi_{*}\right)\right)=0$ in $G_{2 R}^{\sigma / 2}$. Then elliptic $C^{1, \alpha}$-estimates together with (5.4) give the result.

The goal is to prove that $\nabla\left(\psi_{\rho}-\psi_{*}\right)$ is small up to the free boundary of $\psi_{\rho}$. We can prove at least the following:

LEMMA 5.5 Let $R \gg 1$ and $\tau>0$ be fixed. Then there exists $\kappa>0$ with the following property for small $\rho:$ for balls

$$
\begin{gathered}
\hat{B} \subset D_{R} \cap\left\{\psi_{\rho}(\lessgtr) Q_{s}\right\}, \\
\hat{x} \in \partial \hat{B} \cap\left\{\psi_{\rho}=Q_{s}\right\}, \quad
\end{gathered}
$$

we have

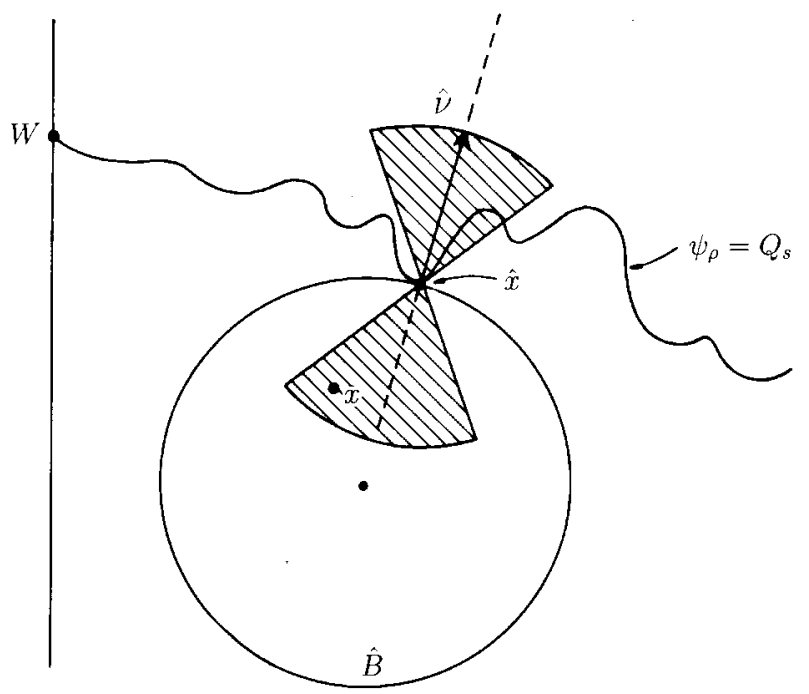

FIG. 20. Cone property.

$$
\nabla \psi_{\rho}(\hat{x}+s e) \cdot e \geqslant \kappa
$$

for $|s| \leqslant 1$ and $|e|=1$ with $e \cdot \hat{v} \geqslant \tau($ resp. $e \cdot(-\hat{v} \geqslant \tau))$, where $\hat{v}$ is the outer normal to $\partial \hat{B}$ at $\hat{x}$.

Proof. It follows from (5.5) that for some $\sigma_{0}>0$ and for small $\rho$, for all $\hat{B}$ as in the statement

$$
\tilde{B}:=\{x \in \hat{B} ;(x-\hat{x}) \cdot \hat{v} \leqslant-1\} \subset G_{R}^{\sigma_{0}} .
$$

Consider the case $\hat{B} \subset\left\{\psi_{\rho}<Q_{s}\right\}$. Then, by (5.4), for small $\rho$

$$
\psi_{\rho} \leqslant \psi_{*}+\varepsilon_{\rho} \leqslant \tilde{\psi}_{*}\left(\omega_{*}-\sigma_{0}\right)+\varepsilon_{\rho} \leqslant Q_{s}-\delta_{0} \quad \text { in } \quad \tilde{B},
$$


for some $\delta_{0}>0$. Since $\psi_{\rho} \leqslant Q_{s}$ and $\nabla \cdot\left(r^{2-n} \nabla \psi_{\rho}\right)=0$ in $\hat{B}$, it follows from elliptic theory that

$$
\psi_{\rho}(x) \leqslant Q_{s}-\kappa_{0} \operatorname{dist}(x, \partial \hat{B}), \quad x \in \hat{B}
$$

for some $\kappa_{0}>0$ being independant of $\rho$ and $\hat{B}$.

Now let $\kappa>0$. Assume the assertion fails. Thus, consider a sequence $\rho \rightarrow 0$ and $\hat{B}_{\rho}, \hat{x}_{\rho}, \hat{v}_{\rho}$ as in the statement and points

$$
\begin{aligned}
x_{\rho} & =\hat{x}_{\rho}+s_{\rho} e_{\rho}, \quad\left|s_{\rho}\right| \leqslant 1, \\
\left|e_{\rho}\right| & =1, e_{\rho} \cdot \hat{v}_{\rho} \geqslant \tau,
\end{aligned}
$$

such that

$$
\nabla \psi_{\rho}\left(x_{\rho}\right) \cdot e_{\rho} \leqslant \kappa
$$

The properties of $\hat{B}_{\rho}$ together with (5.4), (5.5) imply that for a subsequence $\rho \rightarrow 0$

$$
\begin{aligned}
& \hat{x}_{\rho} \rightarrow x_{*}=\left(r_{*}, z_{*}\right)=s_{*} e^{i \omega_{*}} \quad \text { with } s_{*}>0, \\
& \hat{v}_{\rho} \rightarrow v_{*}=i e^{i \omega_{*}} .
\end{aligned}
$$

We claim that $\delta:=\left|x_{\rho}-\hat{x}_{\rho}\right| \rightarrow 0$ as $\rho \rightarrow 0$. If not, it follows from (5.11), (5.8) and (5.5) that for a subsequence $\rho \rightarrow 0, x_{\rho} \in G_{R}^{\sigma}$ for some $\sigma>0$. Then Proposition 5.4 implies, if $s_{\rho} \rightarrow s$ and $e_{\rho} \rightarrow e$ as $\rho \rightarrow 0$

$$
\begin{aligned}
\nabla \psi_{\rho}\left(x_{\rho}\right) \cdot e_{\rho} & \geqslant \nabla \psi_{*}\left(x_{\rho}\right) \cdot e_{\rho}-C(\sigma, R) \varepsilon_{\rho} \\
& \rightarrow \nabla \psi_{*}\left(x_{*}+s e\right) \cdot e
\end{aligned}
$$

Since $\nabla \psi_{*}\left(x_{*}\right)$ is proportional to $\nu *$, the infimum $\kappa_{1}$ for all such values of $s$ and $e$ with $|s| \leqslant$ $1,|e|=1, e \cdot v_{*} \geqslant \tau$ is positive. Thus we derive a contradiction if $\kappa<\kappa_{1}$.

We first consider the case $x_{\rho} \in \hat{B}_{\rho}$, that is $s_{\rho}<0$. We perform the blow-up with respect to the distances $\delta=\left|x_{\rho}-\hat{x}_{\rho}\right|=\left|s_{\rho}\right|$, that is we consider

$$
\varphi_{\delta}(x):=\frac{1}{\delta}\left(\left(\psi_{\rho}-\psi_{*}\right)\left(\hat{x}_{\rho}+\delta x\right)-\left(\psi_{\rho}-\psi_{*}\right)\left(\hat{x}_{\rho}\right)\right) .
$$

The regularity results obtained in Theorems 3.5 and 3.6 apply in a neighbourhood of $x_{*}$, uniformly in $\rho$, to the solutions $\psi_{\rho}$ of (5.3). Therefore the functions $\varphi_{\delta}$ are Lipschitz continuous in any bounded domain, uniformly in $\delta$. We conclude from (5.3) (as in the proof of Lemma 5.2) that $\varphi_{\delta} \rightarrow \varphi$ in $H_{l o c}^{1,2}\left(\mathbb{R}^{2}\right)$ for a subsequence $\delta \rightarrow 0$, and that $\nabla \cdot\left(r_{*}^{2-n} \nabla \varphi\right)=0$; that is, $\varphi$ is harmonic. Moreover, $\varphi$ is globally Lipschitz continuous and $\varphi(0)=0$. Then it follows from Liouville's theorem that $\varphi$ is linear; that is,

$$
\varphi(x)=a \cdot x \text { with } a \in \mathbb{R}^{2} .
$$

For points $\hat{x}_{\rho}+\delta x \in \hat{B}_{\rho}$ we have, using (5.6),

$$
\begin{array}{r}
\varphi_{\delta}(x) \leqslant-\kappa_{0} \operatorname{dist}\left(\hat{x}_{\rho}+\delta x, \partial \hat{B}_{\rho}\right) \\
-\frac{1}{\delta}\left(\psi_{*}\left(\hat{x}_{\rho}+\delta x\right)-\psi_{*}\left(\hat{x}_{\rho}\right)\right)
\end{array}
$$


which, as $\rho \rightarrow 0$, results in lower case,

$$
\varphi(x) \leqslant \kappa_{0} x \cdot v_{*}-\nabla \psi_{*}\left(x_{*}\right) \cdot x \text { for } x \cdot v_{*}<0 .
$$

Since $\nabla \psi_{*}\left(x_{*}\right)=\beta v_{*}$ for some $\beta>0$, we see that (5.9) implies $a=\alpha v_{*}$ with

$$
\alpha \geqslant \kappa_{0}-\beta .
$$

Next we consider a subsequence for which

$$
\frac{1}{\delta}\left(x_{\rho}-\hat{x}_{\rho}\right)=-e_{\rho} \rightarrow-e
$$

with $e \cdot v_{*} \geqslant \tau>0$ and we use (5.9). By (5.5), the free boundary corresponding to $\varphi_{\delta}$ converges to $\left\{x \cdot v_{*}=0\right\}$. It follows from elliptic theory that $\varphi_{\delta} \rightarrow \varphi$ smoothly near $-e$. In particular, $\nabla \varphi_{\delta}\left(-e_{\rho}\right) \rightarrow \nabla \varphi(-e)=\alpha v_{*}$. Using assumption (5.9) we obtain

$$
\begin{aligned}
\nabla \varphi_{\delta}\left(-e_{\rho}\right) \cdot e_{\rho} & =\nabla\left(\psi_{\rho}-\psi_{*}\right)\left(x_{\rho}\right) \cdot e_{\rho} \\
& \leqslant \kappa-\nabla \psi_{*}\left(x_{\rho}\right) \cdot e_{\rho} \\
& \rightarrow \kappa-\beta \nu_{*} \cdot e
\end{aligned}
$$

and find

$$
\kappa \geqslant(\alpha+\beta) \nu_{*} \cdot e \geqslant \tau(\alpha+\beta) \geqslant \tau \kappa_{0},
$$

a contradiction if $\kappa<\tau \kappa_{0}$.

Next we consider the case $s_{\rho}>0$, where we assume that $\psi_{\rho}\left(x_{\rho}\right) \neq Q_{s}$. Again consider the blow-up with respect to $\delta=\left|x_{\rho}-\hat{x}_{\rho}\right|=s_{\rho}$. As before, $\varphi(x)=\alpha x \cdot v_{*}$ with $\alpha \geqslant \kappa_{0}-\beta$. Let $\varepsilon>0$. Then for $x \cdot v_{*} \geqslant \varepsilon$ we have

$$
\begin{aligned}
\frac{1}{\delta}\left(\psi_{\rho}\left(\hat{x}_{\rho}+\delta x\right)-Q_{s}\right) & =\varphi_{\delta}(x)+\frac{1}{\delta}\left(\psi_{*}\left(\hat{x}_{\rho}+\delta x\right)-\psi_{*}\left(\hat{x}_{\rho}\right)\right) \\
& \rightarrow(\alpha+\beta) x \cdot v_{*} \geqslant \kappa_{0} \cdot \varepsilon>0,
\end{aligned}
$$

locally uniformly in $x$. Choosing $\varepsilon<e \cdot v_{*}$ this says that the free boundary corresponding to $\varphi_{\delta}$ stays away from $e$, in particular $\psi_{\rho}\left(x_{\rho}\right)>Q_{s}$ for small $\rho$. We then derive a contradiction as before. Note that a posteriori this proves that $\psi_{\rho}\left(\hat{x}_{\rho}+s e_{\rho}\right)>Q_{s}$ for all $0<s \leqslant 1$.

We are now in a position to prove the following.

THEOREM 5.6 Let e be any direction different from $\pm e^{i \omega_{*}}$. Then for small $\rho$ the free boundary in $\Omega \cap B_{\rho}(W)$ is a graph in direction e.

Proof. Consider the situation for the scaled functions $\psi_{\rho}$. Choose two balls $\hat{B}_{1}, \hat{B}_{2}$ as in Lemma 5.5 and $\hat{W}=(0, \hat{h}) \neq W$, so that a region $G$ as in Fig. 21 is well defined. For definiteness we assume that $\hat{B}_{1}, \hat{B}_{2} \subset\left\{\psi_{\rho}<Q_{s}\right\}$ and that $\hat{h}<h$.

Note, see the previous proof, that $\hat{v}_{1}, \hat{v}_{2}$ are close to $i e^{i \omega_{*}}$ if $\rho$ is small. 


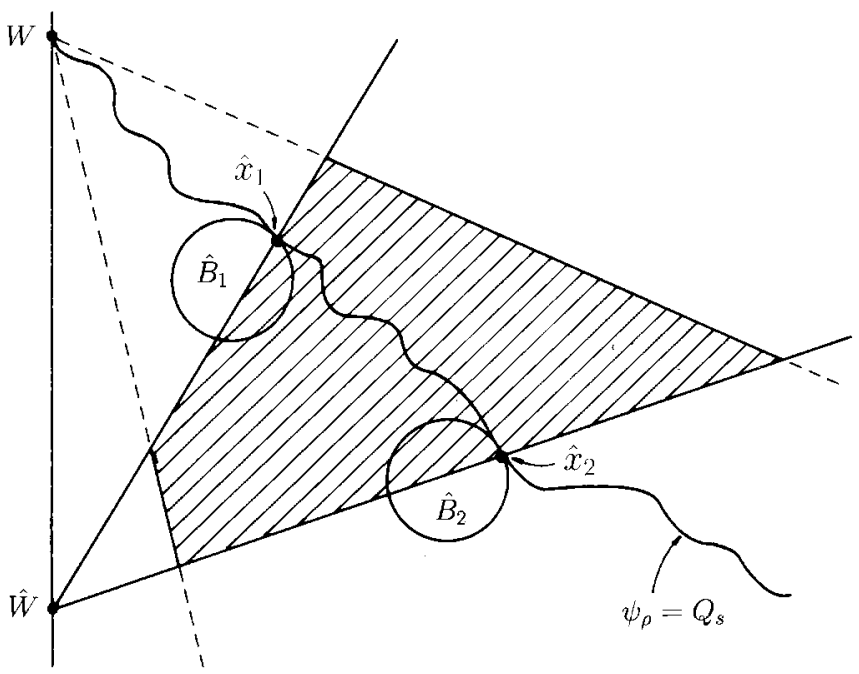

FIG. 21. Shaded region is $G$.

The following is a consequence of (5.4), (5.5) and Lemma 5.5. Consider a ray starting at $\hat{W}$. For rays contributing to $\partial G$, the function $\psi_{\rho}$ is strictly increasing on $\partial G$. For rays cutting $\partial G$, the function $\psi_{\rho}$ attains its minimum at the first cutting point and its maximum at the second cutting point. Now let $\lambda<1$, near 1 , and define

$$
\begin{aligned}
\psi_{\rho \lambda}(x) & :=Q_{s}+\frac{1}{\lambda^{n-1}}\left(\psi_{\rho}(\hat{W}+\lambda(x-\hat{W}))-Q_{s}\right), \\
G_{\lambda} & :=\{x ; \hat{W}+\lambda(x-\hat{W}) \in G\} .
\end{aligned}
$$

Then $\psi_{\rho_{\lambda}}$ is a weak solution in $G_{\lambda}$. Below we show that

$$
\psi_{\rho_{\lambda}}<\psi_{\rho} \text { on } \partial\left(G_{\lambda} \cap G\right) .
$$

If $\psi^{\varepsilon}$ denotes the approximation of $\psi$ from Section 3, then also $\psi_{\rho \lambda}^{\varepsilon}<\psi_{\rho}^{\varepsilon}$ on $\partial\left(G_{\lambda} \cap G\right)$ for small $\varepsilon$. As in Proposition 3.2, it then follows that $\psi_{\rho \lambda}^{\varepsilon} \leqslant \psi_{\rho}^{\varepsilon}$ in $G_{\lambda} \cap G$ resulting in $\psi_{\rho \lambda} \leqslant \psi_{\rho}$ in $G_{\lambda} \cap G$. As a consequence, $\psi_{\rho}$ is monotonically increasing in $G$ along rays starting at $\hat{W}$. Since we can vary $\hat{B}_{1}, \hat{B}_{2}$ and $\hat{W}$ the assertion follows.

It remains to prove (5.13) for $\lambda$ near 1, provided the geometry of $G$ is chosen appropriately. Consider the part

$$
S=\left\{x=\hat{W}+s e ; \quad s_{1} \leqslant s \leqslant s_{2}\right\},
$$

of one of the above rays intersecting $\bar{G}$. It follows from (5.4), (5.5) and Lemma 5.5 that for small $\rho$

$$
\nabla \psi_{\delta}(x) \cdot e \geqslant \kappa
$$


for all $x \in S$ if $S \subset \partial G$, or for $x$ near $\hat{W}+s_{1} e$ and $\hat{W}+s_{2} e$ otherwise. Note that $\kappa>0$ is independent of the domains $G$ that were chosen. It follows that for all points $x=\hat{W}+$ se under consideration and all $\lambda<1$, near 1 ,

$$
\psi_{\rho}(\hat{W}+s e)-\psi_{\rho}(W+\lambda s e) \geqslant \kappa(1-\lambda) s .
$$

Thus with

$$
\begin{aligned}
\xi: & =\psi_{\rho}(\hat{W}+s e), \\
\psi_{\rho \lambda}(\hat{W}+s e)-\psi_{\rho}(\hat{W}+s e) & \leqslant Q_{s}-\xi+\frac{1}{\lambda^{n-1}}\left(\xi-\kappa(1-\lambda) s-Q_{s}\right) \\
& =\frac{1-\lambda}{\lambda^{n-1}}\left(\left(\xi-Q_{s}\right) \frac{1-\lambda^{n-1}}{1-\lambda}-\kappa s\right) \\
& \leqslant \frac{1-\lambda}{\lambda^{n-1}}\left(2 L\left(s_{2}-s_{1}\right)-\kappa s_{1}\right),
\end{aligned}
$$

where $L$ is the Lipschitz constant of $\psi$ in a suitable domain. Then it follows from (5.5) and Proposition 5.4 that for small $\rho$ we can choose in the definition of $G$ the two rays starting at $W$ so that they enclose an angle of magnitude $C \varepsilon_{\rho}$. Finally, we choose $G$ so that $s_{2}-s_{1} \leqslant C \varepsilon_{\rho}$ and $s_{1} \geqslant c>0$. This proves (5.13) for small $\rho$.

\section{Asymptotic behaviour near the well}

In Section 5 we have proved that the free boundary has a tangent $e^{i \omega_{*}}$ at $W$. Now we study how the free boundary approaches this tangent direction. In the analysis we use the standard conformal transformation

$$
r+i(z-h)=e^{s+i \theta}, \quad \rho=e^{s} .
$$

Then the neighbourhood $D:=D_{\rho_{0}}$ in Section 5 becomes

$$
\tilde{D}=\left\{(s, \theta) ;-\frac{\pi}{2}<\theta<\frac{\pi}{2},-\infty<s<s_{0}\right\}, \quad \text { with } s_{0}=\log \rho_{0} .
$$

We denote the transformed functions by a superscript, for instance, $\tilde{\psi}(s, \theta)=\psi(r, z)$ with arguments related by (6.1).

We recall the local weak equation of $\psi$ near $W$ :

$$
\int_{D} \nabla \zeta \cdot\left(\frac{1}{r^{n-2}} \nabla\left(\psi-\psi_{*}\right)+\gamma e_{r}\right) \mathrm{d} r \mathrm{~d} z=0
$$

for all $\zeta \in C_{0}^{\infty}(D)$. Since

$$
\nabla \zeta=\frac{1}{r^{2}+z^{2}}\left[\begin{array}{l}
r-z \\
z-r
\end{array}\right] \nabla \tilde{\zeta}
$$

the transformed weak equation becomes

$$
\int_{\tilde{D}} \nabla \tilde{\zeta} \cdot\left(\frac{1}{r^{n-2}} \nabla\left(\tilde{\psi}-\tilde{\psi}_{*}\right)+\tilde{\gamma}\left[\begin{array}{r}
r \\
-z
\end{array}\right]\right) \mathrm{d} s \mathrm{~d} \theta=0
$$




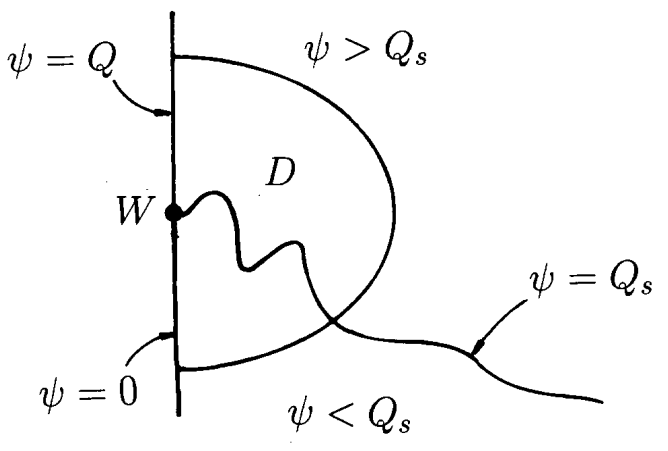

FIG. 22. Free boundary approaching the well.

for all $\tilde{\zeta} \in C_{0}^{\infty}(\tilde{D})$, where $r=e^{s} \cos \theta$ and $z=e^{s} \sin \theta$.

To demonstrate the behaviour near the well we apply the general method of separation of variables by giving an eigenfunction expansion in the $\theta$-direction and by reducing (6.3) to ordinary differential equations for the coefficients in the $s$-variable. We first consider

LEMMA 6.1 The eigenvalue problem, with $m=n-2$ and $n=2$ or 3 ,

$$
\left\{\begin{array}{l}
\partial_{\theta}\left(\frac{1}{\cos ^{m} \theta} \partial_{\theta} u\right)+\frac{1}{\cos ^{m} \theta} \lambda u=0 \quad \text { for }-\frac{\pi}{2}<\theta<\frac{\pi}{2}, \\
u\left(-\frac{\pi}{2}\right)=0, u\left(\frac{\pi}{2}\right)=0,
\end{array}\right.
$$

has the following eigenfunctions $e_{k}$ and eigenvalues $\lambda_{k}$ for $k \geqslant 1$ : for $m=0$

$$
e_{k}(\theta)=\sqrt{\frac{2}{\pi}} \sin \left(k\left(\theta+\frac{\pi}{2}\right)\right), \quad \lambda_{k}=k^{2},
$$

and for $m=1$

$$
e_{k}(\theta)=\left.\sqrt{\frac{\lambda_{k}(2 k+1)}{2}} \frac{1}{2^{k} k !}\left(\partial_{t}^{k-1}\left(t^{2}-1\right)^{k}\right)\right|_{t}=\sin \theta, \quad \lambda_{k}=k(k+1) .
$$

These functions form an orthonormal basis of the weighted $L^{2}$-space with inner product

$$
\langle u, v\rangle:=\int_{-\pi / 2}^{\pi / 2} \frac{u(\theta) v(\theta)}{\cos ^{m}(\theta)} d \theta .
$$

Proof. We only prove the case $m=1$. Consider the transformation $t=\sin \theta$. Then the equation for $\tilde{u}(t):=u(\theta)$ is

$$
\left(1-t^{2}\right) \partial_{t}^{2} \tilde{u}+\lambda \tilde{u}=0
$$


and this gives for $\tilde{v}:=\partial_{t} \tilde{u}$

$$
\partial_{t}\left(\left(1-t^{2}\right) \partial_{t} \tilde{v}\right)+\lambda \tilde{v}=0 .
$$

Solutions $(\tilde{v}, \lambda)$ are given by the Legendre polynomials

$$
P_{k}(t):=\frac{1}{2^{k} k !} \partial_{t}^{k}\left(t^{2}-1\right)^{k}, \quad \lambda_{k}=k(k+1),
$$

normalized so that $P_{k}( \pm 1)=( \pm 1)^{k}$. Moreover,

$$
\int_{-1}^{1} P_{k}(t) P_{\ell}(t) \mathrm{d} t=\frac{2}{2 k+1} \delta_{k, \ell}
$$

Then for $k \geqslant 1$, the functions

$$
\tilde{E}_{k}(t):=\int_{-1}^{t} P_{k}(s) d s=\frac{1}{2^{k} k !} \partial_{t}^{k-1}\left(t^{2}-1\right)^{k}
$$

vanish for $t= \pm 1$. Set $E_{k}(\theta):=\tilde{E}_{k}(\sin \theta)$. Using (6.4) for $\left(\tilde{E}_{k}, \lambda_{k}\right)$ we see that

$$
\begin{aligned}
\left\langle E_{k}, E_{\ell}\right\rangle & =\int_{-1}^{1} \frac{\tilde{E}_{k}(t) \tilde{E}_{\ell}(t)}{1-t^{2}} \mathrm{~d} t=-\frac{1}{\lambda_{k}} \int_{-1}^{1} \tilde{E}_{k}^{\prime \prime}(t) \tilde{E}_{\ell}(t) \mathrm{d} t \\
& =\frac{1}{\lambda_{k}} \int_{-1}^{1} P_{k}(t) P_{\ell}(t) \mathrm{d} t=\frac{2}{\lambda_{k}(2 k+1)} \delta_{k . \ell .}
\end{aligned}
$$

Therefore define $e_{k}:=\sqrt{\frac{\lambda_{k}(2 k+1)}{2}} E_{k}$. The rest of the result then follows from spectral theory.

In addition we need the following estimates.

PROPOSITION 6.2 There exists a constant $C$ so that for all $k \geqslant 1$ and for all $|\theta| \leqslant \frac{\pi}{2}$

$$
\begin{aligned}
& \left|e_{k}(\theta)\right| \leqslant C, \\
& \left|e_{k}^{\prime}(\theta)\right| \leqslant C k^{1+\frac{m}{2}} .
\end{aligned}
$$

Proof. Again we only consider $m=1$. We use the representation

$$
P_{k}(\cos \vartheta)=\sum_{i=o}^{k} b_{i} b_{k-i} \cos ((k-2 i) \vartheta)
$$

where

$$
b_{k}:=\prod_{1 \leqslant i \leqslant k}\left(1-\frac{1}{2 i}\right)=\frac{1 \cdot 3 \cdots(2 k-1)}{2 \cdot 4 \cdots(2 k)}
$$


which implies that for $|t| \leqslant 1$, setting $t=\cos \vartheta$,

$$
\left|P_{k}(t)\right| \leqslant \sum_{i=0}^{k} b_{i} b_{k-i}=P_{k}(\cos 0)=1,
$$

see [10: p93, 290]. It follows, again with $t=\cos \vartheta, 0 \leqslant \vartheta \leqslant \pi$, that

$$
\begin{aligned}
\tilde{E}_{k}(t) & =\int_{\vartheta}^{\pi} P_{k}(\cos \vartheta) \sin \vartheta \mathrm{d} \vartheta \\
& =\sum_{i=0}^{k} \frac{b_{i} b_{k-i}}{2} \int_{\vartheta}^{\pi}\{\sin ((k+1-2 i) \vartheta)-\sin ((k-1-2 i) \vartheta)\} \mathrm{d} \vartheta .
\end{aligned}
$$

With

$$
a_{j}(\vartheta):=j \int_{\vartheta}^{\pi} \sin (j \vartheta) d \vartheta=\cos (j \vartheta)-\cos (j \pi)
$$

this gives

$$
\tilde{E}_{k}(t)=\sum_{\substack{0 \leq i \leqslant k \\ 2 i \neq k+1}} \frac{b_{i} b_{k-i}}{2(k+1-2 i)} a_{k+1-2 i}(\vartheta)-\sum_{\substack{1 \leqslant i \leqslant k+1 \\ 2 i \neq k+1}} \frac{b_{i-1} b_{k+1-i}}{2(k+1-2 i)} a_{k+1-2 i}(\vartheta) .
$$

For $1 \leqslant i \leqslant k$, the expression

$$
\frac{b_{i} b_{k-i}-b_{i-1} b_{k+1-i}}{k+1-2 i}
$$

does not change if we replace $i$ by $k+1-i$, and it equals

$$
\frac{b_{i-1} b_{k-i}}{k+1-2 i}\left(\left(1-\frac{1}{2 i}\right)-\left(1-\frac{1}{2(k+1-i)}\right)\right)=-\frac{b_{i-1} b_{k-i}}{2 i(k+1-i)} .
$$

Further, $a_{j}=a_{-j}$, so that

$$
\tilde{E}_{k}(t)=\frac{b_{0} b_{k}}{k+1} a_{k+1}(\vartheta)-\sum_{1 \leqslant i<\frac{k+1}{2}} \frac{b_{i-1} b_{k-i}}{2 i(k+1-i)} a_{k+1-2 i}(\vartheta) .
$$

Now, $\left|a_{j}(\vartheta)\right| \leqslant 2$ and $b_{k} \leqslant(k+1)^{-1 / 2}$ since

$$
\log b_{k}=\sum_{i=1}^{k} \log \left(1-\frac{1}{2 i}\right) \leqslant-\frac{1}{2} \sum_{i=1}^{k} \frac{1}{i} \leqslant-\frac{1}{2} \log (k+1) .
$$

This implies the estimate

$$
\begin{aligned}
\left|\tilde{E}_{k}(t)\right| & \leqslant 2(k+1)^{-3 / 2}+\sum_{1 \leqslant i<\frac{k+1}{2}} i^{-3 / 2}(k+1-i)^{-3 / 2} \\
& \leqslant 2(k+1)^{-3 / 2}\left(1+\sqrt{2} \sum_{i=1}^{\infty} i^{-3 / 2}\right)
\end{aligned}
$$


which proves (6.5). Since

$$
E_{k}^{\prime}(\theta)=P_{k}(\sin \theta) \cos \theta,
$$

and $\left|P_{k}(\sin \theta)\right| \leqslant 1$, we obtain (6.6).

We note that estimate (6.6) might not be optimal, but it is sufficient to prove the desired convergence of the free boundary. In order to start the procedure, we need the following initial information about the free boundary near the well.

THEOREM 6.3 For small enough $\rho_{0}$ there exists a continuous function $\left.s \mapsto \omega(s) \in\right]-\frac{\pi}{2}, \frac{\pi}{2}[$ so that

1. $\tilde{\psi}(s, \theta)<Q_{s}$, for $-\frac{\pi}{2} \leqslant \theta<\omega(s)$,

$\tilde{\psi}(s, \theta)>Q_{s}$, for $\omega(s)<\theta \leqslant \frac{\pi}{2}$.

2. $\omega(s) \rightarrow \omega_{*}$ as $s \searrow-\infty$,

where $\omega_{*}$ is defined in 5.1.

Proof. Follows from Lemma 5.3 and Theorem 5.6.

Next we define the coefficients in the eigenfunction expansion. For convenience we retain the notation $m=n-2$.

DEFINITION 6.4 For any $s<s_{0}$ and $k \geqslant 1$, set

$$
\begin{aligned}
& \psi_{k}(s):= \int_{-\pi / 2}^{\pi / 2} \frac{e_{k}(\theta)}{\cos ^{m} \theta}\left(\tilde{\psi}(s, \theta)-\tilde{\psi}_{*}(\theta)\right) \mathrm{d} \theta=\left\langle\tilde{\psi}(s, \cdot)-\tilde{\psi}_{*}, e_{k}\right\rangle, \\
& c_{k}(s):= \int_{-\pi / 2}^{\pi / 2} e_{k}(\theta) \tilde{\gamma}(s, \theta) \cos \theta \mathrm{d} \theta=\int_{-\pi / 2}^{\omega(s)} e_{k}(\theta) \cos \theta \mathrm{d} \theta, \\
& s_{k}(s):=\int_{-\pi / 2}^{\pi / 2} e_{k}^{\prime}(\theta) \tilde{\gamma}(s, \theta) \sin \theta \mathrm{d} \theta=\int_{-\pi / 2}^{\omega(s)} e_{k}^{\prime}(\theta) \sin \theta \mathrm{d} \theta .
\end{aligned}
$$

We have the identity

$$
c_{k}(s)+s_{k}(s)=e_{k}(\omega(s)) \sin \omega(s) \quad \text { for all } s<s_{0} \text { and } k \geqslant 1 .
$$

Proposition 6.5 There exists a sequence $\left(s_{j}\right)_{j \in \mathbb{N}}$, with $s_{j} \rightarrow \infty$ as $j \rightarrow \infty$, so that for all $j$ and for all $k \geqslant 1$

$$
\left|\psi_{k}\left(s_{j}\right)\right|^{2}+\left|\psi_{k}^{\prime}\left(s_{j}\right)\right|^{2} \leqslant e^{m s_{j}} .
$$


Proof. By the normalization of $e_{k}$ we have

$$
\left|\psi_{k}(s)\right|^{2} \leqslant \int_{-\pi / 2}^{\pi / 2} \frac{\left|\left(\tilde{\psi}-\tilde{\psi}_{*}\right)(s, \theta)\right|^{2}}{\cos ^{m} \theta} \mathrm{d} \theta .
$$

Since $\left(\tilde{\psi}-\tilde{\psi}_{0}\right)\left(s,-\frac{\pi}{2}\right)=0$ we have

$$
\begin{aligned}
\int_{-\pi / 2}^{0} \frac{\left|\left(\tilde{\psi}-\tilde{\psi}_{*}\right)(s, \theta)\right|^{2}}{\cos ^{m} \theta} d \theta & \leqslant \int_{-\pi / 2}^{0} \frac{\theta+\frac{\pi}{2}}{\cos ^{m} \theta} \int_{-\pi / 2}^{0}\left|\partial_{\theta}\left(\tilde{\psi}-\tilde{\psi}_{*}\right)(s, \tilde{\theta})\right|^{2} \mathrm{~d} \tilde{\theta} \mathrm{d} \theta \\
& \leqslant C \int_{-\pi / 2}^{\pi / 2}\left|\partial_{\theta}\left(\tilde{\psi}-\tilde{\psi}_{*}\right)(s, \theta)\right|^{2} \mathrm{~d} \theta .
\end{aligned}
$$

Similarly we argue for the integral over $[0, \pi / 2]$. Moreover,

$$
\left|\left(\psi_{k}^{\prime}(s)\right)\right|^{2} \leqslant \int_{-\pi / 2}^{\pi / 2} \frac{\left|\partial_{s}\left(\tilde{\psi}-\tilde{\psi}_{*}\right)(s, \theta)\right|^{2}}{\cos ^{m} \theta} \mathrm{d} \theta
$$

so that

$$
\left|\psi_{k}(s)\right|^{2}+\left|\psi_{k}^{\prime}(s)\right|^{2} \leqslant C \int_{-\pi / 2}^{\pi / 2} \frac{\left|\nabla\left(\tilde{\psi}-\tilde{\psi}_{*}\right)(s, \theta)\right|^{2}}{\cos ^{m} \theta} \mathrm{d} \theta .
$$

The smoothness of the boundary data of the difference $\psi-\psi_{*}$ implies $\left|\nabla\left(\psi-\psi_{*}\right)\right|^{2} / r^{m} \in$ $L^{1}(\Omega)$. Consequently,

$$
\begin{gathered}
\infty>\int_{D} \frac{\left|\nabla\left(\psi-\psi_{*}\right)\right|^{2}}{r^{m}} \mathrm{~d} r \mathrm{~d} z=\int_{\tilde{D}} \frac{\left|\nabla\left(\tilde{\psi}-\tilde{\psi}_{*}\right)\right|^{2}}{e^{m s} \cos ^{m} \theta} \mathrm{d} s \mathrm{~d} \theta \\
\geqslant \frac{1}{C} \int_{-\infty}^{s_{0}} e^{-m s} \sup _{k \geqslant 1}\left(\left|\psi_{k}(s)\right|^{2}+\left|\psi_{k}^{\prime}(s)\right|^{2}\right) \mathrm{d} s .
\end{gathered}
$$

From this the assertion follows.

Proposition 6.6 There exists a constant $C$ so that for all $s<s_{0}$ and all $k \geqslant 1$

$$
\begin{aligned}
& \left|s_{k}(s)-s_{k}(-\infty)\right| \leqslant C \\
& \left|s_{1}(s)-s_{1}(-\infty)\right| \leqslant C\left|\omega(s)-\omega_{*}\right| \\
& \left|c_{k}(s)-c_{k}(-\infty)\right| \leqslant C \min \left(\omega(s)-\omega_{*}, k^{\frac{m}{2}-1}\right)
\end{aligned}
$$


Proof. Using identity (6.7) and property (6.5) we see that (6.8) follows from (6.10). Further, (6.9) is obvious since $e_{1}^{\prime}$ is bounded, see (6.6). Also, from property (6.5),

$$
\left|c_{k}(s)-c_{k}(-\infty)\right| \leqslant C\left|\omega(s)-\omega_{*}\right|
$$

therefore it remains to show that for all $-\frac{\pi}{2} \leqslant \theta_{-}<\theta_{+} \leqslant \frac{\pi}{2}$ and for all $k \geqslant 1$

$$
\left|\int_{\theta_{-}}^{\theta_{+}} e_{k}(\theta) \cos \theta \mathrm{d} \theta\right| \leqslant C k^{\frac{m}{2}-1} .
$$

Using the differential equation for $e_{k}$ we obtain

$$
\begin{aligned}
\int_{\theta_{-}}^{\theta_{+}} e_{k}(\theta) \cos \theta \mathrm{d} \theta & =-\frac{1}{\lambda_{k}} \int_{\theta_{-}}^{\theta_{+}}\left(\frac{e_{k}^{\prime}(\theta)}{\cos ^{m} \theta}\right)^{\prime} \cos ^{m+1} \theta \mathrm{d} \theta \\
& =-\frac{1}{\lambda_{k}} e_{k}^{\prime}(\theta) \cos \theta \mid \begin{array}{l}
\theta=\theta_{+} \\
\theta=\theta_{-}
\end{array}+\frac{m+1}{\lambda_{k}} \int_{\theta_{-}}^{\theta_{+}} e_{k}^{\prime}(\theta) \sin \theta \mathrm{d} \theta .
\end{aligned}
$$

The desired estimate follows from property (6.6) and from the observation $\lambda_{k} \geqslant k^{2}$ for all $k \geqslant 1$.

THEOREM 6.7 As $s \searrow-\infty$

$$
\omega(s)-\omega_{*}=c \rho^{1+m} \log \frac{1}{\rho}+O\left(\rho^{1+m}\right),
$$

where $\rho=e^{s}$,

$$
c=\frac{2}{(2+m) Q} \frac{\left|e_{1}\left(\omega_{*}\right)\right|^{2}\left(-\sin \omega_{*}\right)}{\tilde{\varphi}_{*}^{\prime}\left(\omega_{*}\right)}>0,
$$

and

$$
\tilde{\varphi}_{*}^{\prime}\left(\omega_{*}\right)=\left\{\begin{array}{cc}
\frac{2}{\pi} & \text { for } n=2 \\
\cos \omega_{*} & \text { for } n=3
\end{array}\right.
$$

Proof. In the weak equation (6.3) we substitute

$$
\tilde{\zeta}(s, \theta)=\eta(s) e_{k}(\theta) \quad \text { with } \eta \in C_{0}^{\infty}(]-\infty, s_{0}[) .
$$

To evaluate the resulting expression we use the differential equation for $e_{k}$ and Definition 6.4, i.e.

$$
\int_{-\pi / 2}^{\pi / 2} \frac{e_{k}^{\prime}}{\cos ^{m} \theta} \partial_{\theta}\left(\tilde{\psi}-\tilde{\psi}_{*}\right)=\lambda_{k} \psi_{k}(s)
$$


and

$$
\int_{-\pi / 2}^{\pi / 2} \frac{e_{k}}{\cos ^{m} \theta} \partial_{s}\left(\tilde{\psi}-\tilde{\psi}_{*}\right)=\psi_{k}^{\prime}(s) .
$$

Then the weak Eq. (6.3) becomes, with $\rho=e^{s}$,

$$
0=\int_{-\infty}^{s_{0}}\left(\frac{1}{\rho^{m}}\left(\eta^{\prime} \psi_{k}^{\prime}+\eta \lambda_{k} \psi_{k}\right)+\rho\left(\eta^{\prime} c_{k}-\eta s_{k}\right)\right) \mathrm{d} s
$$

for all test functions $\eta$; that is,

$$
\left(\frac{\psi_{k}^{\prime}}{\rho^{m}}+\rho c_{k}\right)^{\prime}=\lambda_{k} \frac{\psi_{k}}{\rho^{m}}-\rho s_{k}
$$

Since $\lambda_{k}=k(k+m)$, this implies the identity

$$
\begin{aligned}
\left(e^{k s}\left(\psi_{k}^{\prime}-(k+m) \psi_{k}+\rho^{m+1} c_{k}\right)\right)^{\prime} & =\left(e^{(k+m) s}\left(\frac{\psi_{k}^{\prime}}{\rho^{m}}+\rho c_{k}\right)-(k+m) e^{k s} \psi_{k}\right)^{\prime} \\
& =e^{k s}\left(\lambda_{k} \psi_{k}-\rho^{m+1} s_{k}\right)+(k+m) e^{k s}\left(\rho^{m+1} c_{k}-k \psi_{k}\right) \\
& =\rho^{k+m+1}\left((k+m) c_{k}-s_{k}\right) .
\end{aligned}
$$

Now we integrate and obtain by (6.10) and Proposition (6.5), using the notation $\tilde{\rho}=e^{\tilde{s}}$,

$$
\psi_{k}^{\prime}-(k+m) \psi_{k}+\rho^{m+1} c_{k}=\rho^{-k} \int_{-\infty}^{s} \tilde{\rho}^{k+m+1}\left((k+m) c_{k}(\tilde{s})-s_{k}(\tilde{s})\right) \mathrm{d} \tilde{s} .
$$

A second integration leads to the formula

$$
\begin{gathered}
\psi_{k}(s)=\left(\frac{\rho}{\rho_{0}}\right)^{k+m} \psi_{k}\left(s_{0}\right)+\rho^{k+m} \int_{s}^{s_{0}} \tilde{\rho}^{1-k} c_{k}(\tilde{s}) \mathrm{d} \tilde{s} \\
-\rho^{k+m} \int_{s}^{s_{0}} \tilde{\rho}^{-2 k-m} \int_{-\infty}^{\tilde{s}} \tilde{\tilde{\rho}}^{k+m+1}\left((k+m) c_{k}(\tilde{\tilde{s}})-s_{k}(\tilde{\tilde{s}})\right) \mathrm{d} \tilde{\tilde{s}} \mathrm{~d} \tilde{s}
\end{gathered}
$$

Let $\psi_{k}^{0}(s)$ be the same expression, except that $c_{k}$ is replaced by $c_{k}^{0}:=c_{k}(-\infty)$ and $s_{k}$ by $s_{k}^{0}:=$ $s_{k}(-\infty)$. Then a computation using (6.7) gives

$$
\psi_{k}^{0}(s)=\left(\frac{\rho}{\rho_{0}}\right)^{k+m} \psi_{k}\left(s_{0}\right)+\frac{e_{k}\left(\omega_{*}\right) \sin \omega_{*}}{k+m+1} \varphi_{k}^{0}(s)
$$

where

$$
\varphi_{k}^{0}(s)=\rho^{k+m}\left\{\begin{array}{cl}
s_{0}-s & \text { for } k=1 \\
\frac{1}{k-1}\left(\rho^{1-k}-\rho_{0}^{1-k}\right) & \text { for } k \geqslant 2 .
\end{array}\right.
$$


Now, since $\left\{e_{k} ; k \geqslant 1\right\}$ is an orthonormal system in the Hilbert space defined in Lemma 6.1, we have for all $s$ and for almost all $\theta$ the representation

$$
\tilde{\psi}(s, \theta)-\tilde{\psi}_{*}(\theta)=\sum_{k=1}^{\infty} \psi_{k}(s) e_{k}(\theta) .
$$

Let us evaluate the left-hand side at the free boundary, that is for $\theta=\omega(s)$. Using the identity for $\omega_{*}$ from Theorem 6.3, we obtain

$$
\begin{aligned}
\tilde{\psi}(s, \omega(s))-\tilde{\psi}_{*}(\omega(s)) & =Q_{s}-\tilde{\psi}_{*}(\omega(s)) \\
& =\tilde{\psi}_{*}\left(\omega_{*}\right)-\tilde{\psi}_{*}(\omega(s)) \\
& =\frac{Q}{2}\left(\tilde{\varphi}_{*}\left(\omega_{*}\right)-\tilde{\varphi}_{*}(\omega(s))\right) .
\end{aligned}
$$

Since $\omega(s) \rightarrow \omega_{*}$ as $s \searrow-\infty$ (see Theorem 6.3, second statement) the right-hand side of this equality can be expanded. This results in

$$
\tilde{\psi}(s, \omega(s))-\tilde{\psi}_{*}(\omega(s))=-\frac{Q}{2} \tilde{\varphi}_{*}^{\prime}\left(\omega_{*}\right)\left(\omega(s)-\omega_{*}\right)+O\left(\left|\omega(s)-\omega_{*}\right|^{2}\right) .
$$

The goal is to prove from these identities, that the behaviour of the free boundary near the well, that is the first term in the expansion of $\omega(s)-\omega_{*}$ as $s \searrow-\infty$, is given by $\psi_{1}^{0}(s)$.

For this we first use the results from Proposition 6.6 and obtain for $k \geqslant 2$ the estimate

$$
\left|\psi_{k}(s)-\psi_{k}^{0}(s)\right| \leqslant C k^{\frac{m}{2}-1} \varphi_{k}^{0}(s) \leqslant C \rho^{1+m} k^{\frac{m}{2}-2} .
$$

Here, and in the following estimates, the constants $C$ do not depend on $\theta$ and $s_{0}$. Using (6.5) we obtain

$$
\left|\sum_{k \geqslant 2}\left(\psi_{k}(s)-\psi_{k}^{0}(s)\right) e_{k}(\theta)\right| \leqslant C \rho^{1+m},
$$

and also

$$
\left|\sum_{k \geqslant 2}\left(\psi_{k}^{0}(s)-\left(\frac{\rho}{\rho_{0}}\right)^{k+m} \psi_{k}\left(s_{0}\right)\right) e_{k}(\theta)\right| \leqslant C \rho^{1+m} .
$$

All computations also hold if we replace $s_{0}$ by a smaller value. Let us replace $s_{0}$ by one of the values $\left(s_{j}\right)_{j \in \mathbb{N}}$ from Proposition 6.5 and let $\rho_{j}=e^{s_{j}}$. Note that now all $\psi_{k}^{0}$ depend on $j$. Then for $s<s_{j}$

$$
\left|\sum_{k \geqslant 1}\left(\frac{\rho}{\rho_{j}}\right)^{k+m} \psi_{k}\left(s_{j}\right) e_{k}(\theta)\right| \leqslant C \sum_{k \geqslant 1} \frac{\rho^{k+m}}{\rho_{j}^{k+\frac{m}{2}}}=C \frac{\rho^{1+m}}{\left(\rho_{j}-\rho\right) \rho_{j}^{\frac{m}{2}}} .
$$

Altogether we obtain from (6.12)

$$
\begin{gathered}
\tilde{\psi}(s, \theta)-\tilde{\psi}_{*}(\theta)=\left(\psi_{1}(s)-\psi_{1}^{0}(s)\right) e_{1}(\theta)+ \\
\frac{e_{1}\left(\omega_{*}\right) \sin \omega_{*}}{m+2} \varphi_{1}^{0}(s) e_{1}(\theta)+O\left(\rho^{1+m}\left(1+\frac{1}{\left(\rho_{j}-\rho\right) \rho_{j}^{\frac{m}{2}}}\right)\right) .
\end{gathered}
$$


Letting $\theta \rightarrow \omega(s)$ we obtain using (6.13) and restricting arguments to $s \leqslant s_{j}-1$

$$
\begin{aligned}
\frac{Q}{2}\left(\tilde{\varphi}_{*}\left(\omega_{*}\right)-\tilde{\varphi}_{*}(\omega(s))\right)= & \frac{e_{1}\left(\omega_{*}\right) \sin \omega_{*}}{m+2} \varphi_{1}^{0}(s) e_{1}(\omega(s)) \\
& +\left(\psi_{1}(s)-\psi_{1}^{0}(s)\right) e_{1}(\omega(s))+O\left(\rho^{1+m}\left(1+\frac{1}{\rho_{j}^{1+\frac{m}{2}}}\right)\right) .
\end{aligned}
$$

Using again (6.5) and Proposition 6.6 we see that

$$
\begin{gathered}
\left|\left(\psi_{1}(s)-\psi_{1}^{0}(s)\right) e_{1}(\omega(s))\right| \\
\leqslant C \rho^{1+m} \int_{s}^{s_{j}}\left(\left|\omega(\tilde{s})-\omega_{*}\right|+\tilde{\rho}^{-2-m} \int_{-\infty}^{\tilde{s}} \tilde{\tilde{\rho}}^{2+m}\left|\omega(\tilde{\tilde{s}})-\omega_{*}\right| \mathrm{d} \tilde{\tilde{s}}\right) \mathrm{d} \tilde{s},
\end{gathered}
$$

which gives the rough estimate

$$
\left|\left(\psi_{1}(s)-\psi_{1}^{0}(s)\right)\right| \leqslant C \rho^{1+m}\left(s_{j}-s\right)
$$

Now fix $j=j_{0}$. Then for large negative values of $s$, say $s \leqslant s_{*}<s_{j_{0}}$, the left-hand side of (6.14) is estimated by

$$
\geqslant c\left|\omega(s)-\omega_{*}\right|
$$

Therefore we obtain from (6.14), (6.16) for such values of $s$

$$
\begin{aligned}
\left|\omega(s)-\omega_{*}\right| & \leqslant C \rho^{1+m}\left(1+\frac{1}{\rho_{j_{0}}^{1+\frac{m}{2}}}+\left(s_{j_{0}}-s\right)\right) \\
& \leqslant C\left(s_{j_{0}}, s_{*}, \varepsilon\right) \rho^{1+m-\varepsilon}
\end{aligned}
$$

for $\varepsilon>0$. Now let $\varepsilon=\frac{1}{2}$ and choose $j$ with $s_{j} \leqslant s_{*}$. Then we obtain from (6.14) for $s \leqslant s_{j}$

$$
\left|\psi_{1}(s)-\psi_{1}^{0}(s) e_{1}(\omega(s))\right| \leqslant C\left(s_{j_{0}}, s_{*}, s_{j}\right) \rho^{1+m},
$$

so that from (6.14)

$$
\left|\frac{Q}{2}\left(\tilde{\varphi}_{*}(\omega(s))-\tilde{\varphi}_{*}\left(\omega_{*}\right)\right)+\frac{\left(e_{1}\left(\omega_{*}\right)\right)^{2} \sin \omega_{*}}{m+2} \rho^{1+m}\left(s_{j}-s\right)\right| \leqslant C_{1}\left(s_{j_{0}}, s_{*}, s_{j}\right) \rho^{1+m} .
$$

From this the assertion follows.

\section{The vanishing $Q_{s}$ limit}

In this section we show that for $Q$ sufficiently small, the limit $Q_{s} \searrow 0$ leads to an interface with singular behaviour at the central axis. This limit interface will be at a positive distance below the well. 
To explain the meaning of singular behaviour, we first recall some definitions and results from [5] in the context of the axial symmetric domain $\tilde{\Omega}$ (see Section 2). In that paper we studied the case $Q_{s}=0$ directly; i.e. we considered the case of stagnant salt water underlying fresh water flowing towards the well $W$, while the height of the interface along the cylindrical lateral boundary was fixed at a distance $h-u_{0}$ below the well. This yields a one-phase free boundary problem in terms of the variable

$$
\tilde{w}=\left\{\begin{array}{lll}
\tilde{p}+x_{n} & \text { in } & \tilde{\Omega}_{f}, \\
0 & \text { in } & \tilde{\Omega}_{s},
\end{array}\right.
$$

where $\tilde{p}$ denotes the fluid pressure (as in the proof of Theorem 3.5).

Given a distance $h-u_{0}$, it was shown that a maximal rate exists, the so-called critical rate $Q_{c r}=Q_{c r}\left(h-u_{0}\right)$, such that only for $\left.Q \leqslant Q_{c r}\left(h-u_{0}\right)\right)$ could the existence of $\tilde{w}$ in an appropriate setting be established. In the same range, the corresponding free boundary has a positive distance from the well and is the graph in vertical direction of a function of the horizontal coordinates. The free boundary conditions are (at points of sufficient smoothness)

$$
\tilde{w}=0 \quad \text { and } \quad \partial_{\nu} \tilde{w}=e_{n} \cdot v \quad \text { at the free boundary. }
$$

Moreover, if $Q<Q_{c r}\left(h-u_{0}\right)$, then $\tilde{w}>0$ in an upper neighbourhood of the free boundary which is then analytic. As explained in [5], smoothness of the free boundary is implied by positivity of $\tilde{w}$ in an upper neighbourhood of the free boundary and vice versa.

Uniqueness was also established for subcritical rates, implying that $\tilde{w}$ and the free boundary are axial symmetric (at least in the context of this paper).

The case $Q=Q_{c r}\left(h-u_{0}\right)$ was considered as the limit $Q \nearrow Q_{c r}$. As a result we established the existence of an axial symmetric free boundary (and $\tilde{w}$ ), which loses regularity at the central axis: i.e. points where $\tilde{w}<0$ converge from above to the free boundary at the central axis. In [6] we studied the consequence of this behaviour when $n=2$ in detail, leading to the formation of a cusp in the free boundary.

Because we treat here the cases $n=2,3$ together, we say that a cusp is formed at the free boundary whenever points with $\tilde{w}<0$ enter the free boundary: see Property 4.17 of [5] for the precise statement.

We start by showing some monotonicity results for the weak formulation. They follow directly from Proposition 3.2 and are only valid for solutions constructed according to the procedure of Section 3, that is to say if they are 'constructed accordingly'. We show monotonicity with respect to $Q$ for fixed $Q_{s}$, and with respect to $Q_{s}$ for fixed $Q$.

LEMMA 7.1 Let $Q_{1}, Q_{2}$ denote total discharges satisfying $Q_{1}>Q_{2}>Q_{s}$ (for $Q_{s}$ fixed) and let $Q_{s_{1}}, Q_{s_{2}}$ denote salt discharges satisfying $Q_{s_{1}}<Q_{s_{2}}<Q$ (for $Q$ fixed). If $g_{1}$ and $g_{2}$ denote the free boundaries of the correspondingly constructed weak solutions for one of these pairs of ordered data, then

$$
g_{1}(r) \leqslant g_{2}(r) \quad \text { for all } r \in[0, R] .
$$

Proof. The function $\varphi:=\psi-Q_{s}$ satisfies the weak formulation $(*)$ and (**) with $Q_{s}=0$ and with the modified Dirichlet data, see Fig. 7,

$$
\varphi=\left\{\begin{array}{ccc}
-Q_{s} & \text { on } & A O W \\
Q-Q_{s} & \text { on } & B C W
\end{array}\right.
$$


For both pairs of Dirichlet data we have $\varphi_{1} \geqslant \varphi_{2}$ on $\partial \Omega_{D}$. The same ordering carries over to the Dirichlet data in $B C_{\varepsilon}$ and, by Proposition 3.2, to the approximations $\varphi_{i_{\varepsilon}}$. Passing to the limit gives $\varphi_{1} \geqslant \varphi_{2}$ in $\bar{\Omega}$. Identifying the free boundaries with the level sets $\left\{\varphi_{i}=0\right\}$ and using the $z$-monotonicity of $\varphi_{2}$, yields the inequality.

Next we turn to the convergence for vanishing $Q_{s}$. For convenience we denote the weak solution coresponding to the pair $\left(Q_{s}, Q\right)$ by $\psi_{s}$.

The main result is

THEOREM 7.2 Let $Q<Q_{c r}(h)$ be fixed. Then

$$
\left.\psi:=\lim _{Q_{s} \searrow 0} \psi_{s} \text { ( exists in } \bar{\Omega} \backslash W\right)
$$

is a weak solution corresponding to $Q_{s}=0$. The corresponding free boundary has a cusp at the central axis. In other words, $\psi$ is a cusp solution corresponding to $u_{0}:=\lim _{Q_{s} \searrow 0} u_{s}$.

Proof. As in Lemma 2.1 we only give the proof for $n=3$. We first constuct a comparison function for the solutions $\psi_{s}$ to ensure that for all $Q_{s}>0$, the free boundary stays away from the bottom of $\Omega$. Choose any $\left.Q_{c} \in\right] Q, Q_{c r}(h)\left[\right.$ and let $\tilde{w}_{c}$ denote the subcritical solution related to $Q_{c}$ and $u_{0}=0$, see Fig. 23 .

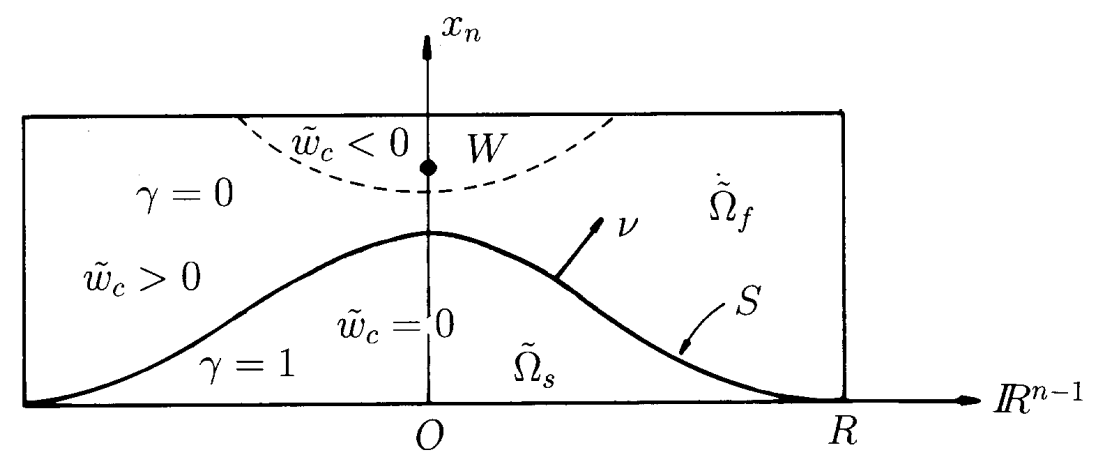

FIG. 23. Properties of the subcritical solution $\tilde{w}_{c}$.

Since subcritical solutions have smooth free boundaries, we find that $\tilde{w}_{c}$ satisfies

$$
\begin{cases}\Delta \tilde{w}_{c}=2 \pi Q_{c} \delta_{W} & \text { in } \tilde{\Omega}_{f} ; \\ \partial_{n} \tilde{w}_{c}=0 & \text { along top; } \\ \tilde{w}_{c}=z & \text { along lateral boundary; } \\ \tilde{w}_{c}=0, \partial_{\nu} \tilde{w}_{c}=e_{n} \cdot v & \text { along } S ; \\ \tilde{w}_{c}=0 & \text { in } \tilde{\Omega}_{s} .\end{cases}
$$

Since $\tilde{w}_{c}$ is radial symmetric we define, as before, the two-dimensional pressure

$$
p_{c}(r, z)=w_{c}(r, z)-z \text { in } \Omega_{f},
$$


and through relations (2.5) a stream function $\psi_{f_{c}}: \bar{\Omega}_{f} \backslash W \rightarrow \mathbb{R}$. Following the proof of Lemma 2.1, we choose $\psi_{f_{c}}$ to be the solution of (see Fig. 24),

$$
\left\{\begin{array}{lll}
\nabla \cdot\left(\frac{1}{r} \nabla \psi\right)=0 & \text { in } & \Omega_{f} \\
\psi=Q_{s} & \text { on } & S \cup T W \\
\psi=Q_{s}+Q_{c} & \text { on } & B C W \\
\partial_{r} \psi=0 & \text { on } & A B .
\end{array}\right.
$$

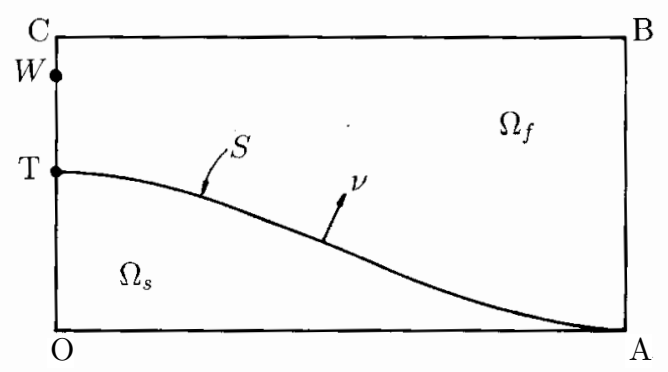

FIG. 24. Definition of $\psi_{f_{c}}$.

One easily verifies that $w=0$ along $S$ implies the second condition

$$
\frac{1}{r} \partial_{\nu} \psi_{f_{c}}+e_{r} \cdot v=0 \quad \text { on } \quad S
$$

Next we extend the construction below the free boundary. Let $\psi_{s_{c}}: \bar{\Omega}_{s} \rightarrow \mathbb{R}$ be the solution of

$$
\begin{cases}\nabla \cdot\left(\frac{1}{r} \nabla \psi\right)=0 & \text { in } \quad \Omega_{s} ; \\ \psi=Q_{s} & \text { on } \quad S ; \\ \psi=\psi_{B} & \text { on } \quad A O T .\end{cases}
$$

where $\psi_{B}$ is a smooth function satisfying $0<\psi_{B} \leqslant Q_{s}, \psi_{B}(A)=\psi_{B}(T)=Q_{s}$ and $\psi_{B} \not \equiv Q_{s}$. Such a $\psi_{s_{c}}$ clearly exists and satisfies

$$
\frac{1}{r} \partial_{\nu} \psi_{s_{c}}>0 \quad \text { on } \quad S
$$

We now show that the composite function $\psi_{c}: \bar{\Omega} \rightarrow \mathbb{R}$, defined by

$$
\psi_{c}=\left\{\begin{array}{lll}
\psi_{f_{c}} & \text { in } & \Omega_{f}, \\
\psi_{s_{c}} & \text { in } & \Omega_{s},
\end{array}\right.
$$


is a supersolution for $\psi_{s}$, for any $Q_{s}>0$. To see this we extend $\psi_{c}$ by $\psi_{c}=Q_{s}+Q_{c}$ in the half strip $\{(r, z): 0 \leqslant r \leqslant R, z \geqslant 1\}$. We shift the composite function downwards over distance $L$ to obtain

$$
\psi_{c_{L}}(r, z)=\psi_{c}(r, z+L) \quad(r, z) \in \bar{\Omega} .
$$

Since $Q_{s}+Q_{c}>Q$ we have

$$
\psi_{c_{L}}>\psi_{s} \text { in } \bar{\Omega},
$$

for $L$ sufficiently large and for all $Q_{s}>0$. Next we decrease $L$, i.e. shift $\psi_{c}$ upwards, until the two functions touch. We claim that this cannot happen for any $L \geqslant 0$.

Since

$$
\left[\frac{1}{r} \partial_{\nu} \psi_{c_{L}}\right]+e_{r} \cdot v<0 \quad \text { on } S,
$$

it follows from the Comparison Lemma 5.2 that the functions cannot touch in interior points of $\Omega$. Obviously, not on $\partial_{D} \Omega$ and, by the strong maximum principle, not on $\partial_{N} \Omega$. The latter observation follows from the fact that $\psi_{c_{L}}>Q_{s}$ on $\partial_{N} \Omega$ for all $L \geqslant 0$.

Denoting the free boundaries of $\psi_{s}, w_{c}$ by the functions $g_{s}, u_{c}$ (see Theorem 4.5) we deduce from the comparison that for all $Q_{s}>0$

$$
g_{s}(r) \geqslant u_{c}(r)>0 \quad \text { on } \quad[0, R[,
$$

and, in particular,

$$
u_{s} \geqslant 0 .
$$

As a second step we consider the convergence of $\psi_{s}$. As in the existence proof of Section 3 we deduce the uniform estimate

$$
\int_{\Omega} \frac{1}{r}\left|\nabla\left(\psi_{s}-\psi_{0}\right)\right|^{2} \leqslant C .
$$

Since $\psi_{0}$ does not depend on $Q_{s}$, we obtain for a sequence $Q_{s} \searrow 0$ :

$$
\begin{aligned}
& \psi_{s}-\psi_{0} \rightarrow \psi-\psi_{0} \text { weakly in } H^{1,2}(\Omega) ; \\
& \psi_{s}-\psi_{0} \rightarrow \psi-\psi_{0} \text { strongly in } L^{2}(\Omega) \text { and a.e. in } \Omega ; \\
& \gamma_{s}:=\chi_{\left\{\psi_{s}<Q_{s}\right\}} \rightarrow \gamma \text { weak star in } L^{\infty}(\Omega) ; \\
& \gamma_{N_{s}}:=\chi_{\left\{\psi_{s}<Q_{s}\right\}} \rightarrow \gamma_{N} \text { weak star in } L^{\infty}\left(\partial_{N} \Omega\right) .
\end{aligned}
$$

The triple $\left\{\psi, \gamma, \gamma_{N}\right\}$ satisfies $(\star)$ and $(\star \star)$ for $Q_{s}=0$,

$$
0 \leqslant \psi<Q \text { in } \Omega,
$$

and (in sense of distributions in $\Omega$ )

$$
\begin{aligned}
& \nabla \cdot\left(\frac{1}{r} \nabla \psi+\gamma e_{r}\right)=0 ; \\
& \partial_{z} \psi \geqslant 0 \text { (inherited from approximations); } \\
& \partial_{r} \gamma \leqslant 0 \text { (from weak equation and } \psi \geqslant 0 \text { ). }
\end{aligned}
$$


Let $\partial\{\psi>0\} \cap \Omega$ denote the free boundary of the limit problem. The $z$-monotonicity of $\psi$ and $r$-monotonicity of $\gamma$ (apply same argument as in the proof of Claim 4.4) imply for $\psi$ the property: if $\psi\left(r_{0}, z_{0}\right)>0$ for some $\left(r_{0}, z_{0}\right) \in \Omega$, then $\psi>0$ in the set $\left\{(r . z): r \geqslant r_{0}, z \geqslant z_{0}\right\}$. This tells us that the free boundary is a Lipschitz graph in any intermediate direction between $e_{r}$ end $e_{z}$, that it has well-defined end points $\left(0, u_{T}\right)$ and $\left(R, u_{0}\right)$, and that it is decreasing it $r$. In fact it is strictly decreasing. The occurrence of a horizontal segment would lead to a contradiction using the Hopf principle and the free boundary condition $\partial_{\nu} \psi=0$.

According to Lemma 7.1, the free boundaries of the approximating problems decrease with $Q_{s} \searrow 0$. From this monotonicity and $\partial_{r} \gamma_{s} \leqslant 0$ one deduces that a mushy region, where $\psi=0$ and $\gamma<1$, cannot exist. Hence

$$
\gamma=1 \quad \text { in } \quad \Omega \backslash \overline{\{\psi>0\}}
$$

and

In other words

$$
\gamma_{N}=1 \quad \text { in } \quad \partial_{N} \Omega \backslash \overline{\{\psi>0\}}
$$

$$
\gamma=1-\chi_{\{\psi>0\}} \quad \text { in } \quad \Omega \quad \text { and } \quad \gamma_{N}=1-\chi_{\{\psi>0\}} \quad \text { in } \quad \partial_{N} \Omega .
$$

Furthermore, there exists $g \in C([0, R]), g$ strictly decreasing and $g(r) \geqslant u_{c}(r)>0$ on $[0, R[$, such that

$$
\overline{\partial\{\psi>0\} \cap \Omega}=\{(r, z): 0 \leqslant r \leqslant R, z=g(r)\} .
$$

We only need to verify the continuity. Using the monotonicity, this follows directly if we can rule our vertical segments. This is done by a similar argument as in the proof of Proposition 4.2. As in Remark 4.6 it follows that

$$
\partial_{r} \psi=0 \text { in }\left\{(R, z): u_{0}<z<1\right\} .
$$

In the third step we return to the function $\tilde{w}$, defined in (7.1). First find the two-dimensional pressure $p \in H_{l o c}^{1,2}(\Omega) \cap C(\Omega)$ from the definitions

$$
\left.\begin{array}{l}
\partial_{z} p=-\frac{1}{r} \partial_{r} \psi-\gamma \\
\partial_{r} p=+\frac{1}{r} \partial_{z} \psi
\end{array}\right\} \text { in } \Omega
$$

such that $p=0$ along the bottom of $\Omega$. Then set

$$
w=p+z \quad \text { in } \Omega .
$$

The weak equation for $\psi$ implies for $w$ the weak equation

$$
\nabla \cdot\left(r\left(\nabla w-\chi_{\{\psi>0\}} e_{z}\right)\right)=0 \quad \text { in } \quad \Omega .
$$

Introducing $\tilde{w}_{0}: \tilde{\Omega} \backslash W \rightarrow \mathbb{R}$ as the unique solution of

$$
\begin{gathered}
\Delta \tilde{w}_{0}=2 \pi Q \delta_{W} \text { in } \tilde{\Omega}, \\
\tilde{B C} \begin{cases}\partial_{z} \tilde{w}_{0}=0 & \text { on the top, } \\
\tilde{w}_{0}=0 & \text { on the bottom, } \\
\tilde{w}_{0}=\left(z-u_{0}\right)_{+} & \text {on the lateral sides, }\end{cases}
\end{gathered}
$$


we find for $\tilde{w}-\tilde{w}_{0}$ the weak equation

$$
\nabla \cdot\left(\nabla\left(\tilde{w}-\tilde{w}_{0}\right)+\tilde{\gamma}\right)=0 \quad \text { in } \quad \tilde{\Omega}
$$

with

$$
\begin{array}{rlrl}
\tilde{w} & =0, & \tilde{\gamma} & =1 \\
\Delta \tilde{w} & =0, & & \text { below free boundary, } \\
& =0 & \text { above free boundary. }
\end{array}
$$

Moreover, $\tilde{w}$ satisfies $\tilde{B C}$, which follows directly from definitions (7.4), (7.5) and the $\psi$-boundary conditions, including (7.3).

These properties imply that $(\tilde{w}, \tilde{\gamma})$ is a weak solution of the free boundary problem introduced in [5]. There it was shown that if, for some open set $U \subset \overline{\tilde{\Omega}} \cap\left\{x_{3}=0\right\}$ and for some $\varepsilon>0$,

$$
\tilde{w}>0 \quad \text { in } \quad\left\{\left(x_{1}, x_{2}, x_{3}\right):\left(x_{1} \cdot x_{2}\right) \in U, \tilde{g}<x_{3}<\tilde{g}+\varepsilon\right\}
$$

then

$$
\tilde{g} \text { is analytic in } U \text {. }
$$

The monotonicity of the free boundary, combined with

$$
\partial_{r} w \geqslant 0 \text { in } \Omega, \text { in sense of distributions, }
$$

which is a consequence of the $z$-monotonicity of $\psi$, imply that $\tilde{w}>0$ in an upper neighbourhood of the free boundary, away from the central axis. Hence

$$
g \text { is analytic in }] 0, R[\text {. }
$$

To conclude the proof, we show that negativity of $\tilde{w}$ enters the free boundary at the top $\left(0, u_{T}\right)$. Clearly, $\tilde{w}<0$ near the well. This follows from the observation that $\tilde{w}_{0} \rightarrow-\infty$ when approaching $W$ and $\tilde{w}-\tilde{w}_{0} \in C^{\alpha}(\tilde{\Omega})$ for some $\alpha \in(0,1)$. Consequently, the free boundary for $Q_{s}=0$ has a positive distance from the well: i.e.

$$
u_{T}<h .
$$

For $Q_{s}>0$ we define the function $w_{s}$ (and $\tilde{w}_{s}$ ) from (7.4), (7.5), with $\psi=\psi_{s}$ and $\gamma=\gamma_{s}$, such that $w_{s}(O)=0$. We first show that $w_{s}$ is non-decreasing along the free boundary: i.e.

$$
\left.w_{s}\left(r, g_{s}(r)\right) \text { is non-decreasing in } r \in\right] 0, R[\text {. }
$$

If the free boundary were smooth, this would be a direct consequence of the Hopf principle, applied to $\psi_{s}$ in $\left\{\psi_{s}<Q_{s}\right\}$, and transformations (7.4), (7.5). These transformations imply

$$
\begin{cases}\frac{1}{r} \nabla \psi_{s}-e_{r}=\left(-\partial_{z} w_{s}, \partial_{r} w_{s}\right) & \text { in } \quad \Omega_{f}, \\ \frac{1}{r} \nabla \psi_{s}=\left(-\partial_{z} w_{s}, \partial_{r} w_{s}\right) & \text { in } \Omega_{s} .\end{cases}
$$

Hence, for an orientation as below we find along the free boundary 


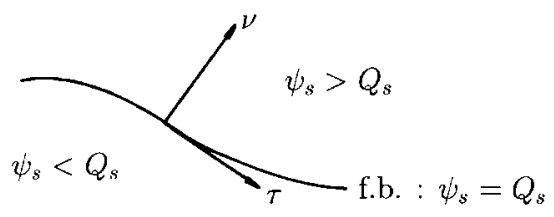

$$
\frac{1}{r} \partial_{\nu}^{+} \psi_{s}-e_{r} \cdot v=v \cdot\left(-\partial_{z} w_{s}, \partial_{r} w_{s}\right)=\nabla w_{s} \cdot\left(v_{z},-v_{r}\right)=\partial_{\tau} w_{s}
$$

and, reflecting the free boundary conditions,

$$
0<\partial_{v}^{-} \psi_{s}=\partial_{\tau} w_{s} \quad \text { ( Hopf). }
$$

Since we established only continuity for the free boundary we have to argue in a different way. The starting point is the weak equation for $\psi_{s}$ : for all $\zeta \in C_{0}^{\infty}(\Omega)$

$$
\begin{aligned}
0 & =\int_{\Omega} \nabla \zeta \cdot\left(\frac{1}{r} \nabla \psi_{s}+\gamma_{s} e_{r}\right) \\
& =\int_{\Omega \cap\left\{\psi_{s}>Q_{s}\right\}} \nabla \zeta \cdot\left(\frac{1}{r} \nabla \psi_{s}-e_{r}\right)+\int_{\Omega} \nabla \zeta \cdot \frac{1}{r} \nabla \min \left(\psi_{s}, Q_{s}\right),
\end{aligned}
$$

where we replaced $\gamma_{s}$ by $-\left(1-\gamma_{s}\right)=-\chi_{\left\{\psi_{s}>Q_{s}\right\}}$. Since $\min \left(\psi_{s}, Q_{s}\right)$ is a supersolution, the second integral is non-negative for $\zeta \geqslant 0$. Substituting (7.9) yields

$$
0 \geqslant \int_{\Omega \cap\left\{\psi_{s}>Q_{s}\right\}} \nabla \zeta \cdot\left(-\partial_{z} w_{s}, \partial_{r} w_{s}\right) \quad \text { for all } \zeta \in C_{0}^{\infty}(\Omega), \zeta \geqslant 0 .
$$

Let $x_{\kappa}=\left(r_{\kappa}, z_{\kappa}\right) \in\left\{\psi_{s}=Q_{s}\right\}, k=1,2$ and $r_{1}<r_{2}$, be two free boundary points and let $R \subset \subset \Omega$ denote a rectangle as in Fig. 25. Further, let the non-negative test functions $\zeta$ convergence towards the characteristic function of $R$. Since $w \in H_{l o c}^{1,2}(\Omega) \cap C(\Omega)$, it follows that for almost all such $R$

$$
0 \geqslant \int_{\partial R \cap\left\{\psi_{s}>Q_{s}\right\}}(-v) \cdot\left(-\partial_{z} w_{s}, \partial_{r} w_{s}\right)=-\int_{\partial R \cap\left\{\psi_{s}>Q_{s}\right\}} \partial_{\tau} w_{s}=w_{s}\left(x_{1}\right)-w_{s}\left(x_{2}\right) .
$$

Because $w$ is continuous, this establishes (7.8). Finally, we consider again the limit $Q_{s} \searrow 0$, now for the functions $\tilde{w}_{s}$. Since $\left(\tilde{w}_{s}, \tilde{\gamma}_{s}\right)$ satisfies (7.6) and $\tilde{w}_{s}-\tilde{w}_{0}=0$ (by appropriate normalization) along the lateral boundary of $\tilde{\Omega}_{f}$, we have $H^{1,2}$ and $C^{\alpha}$ estimates implying for a sequence $Q_{s} \searrow 0$,

$$
\tilde{w}_{s}-\tilde{w}_{0} \rightarrow \tilde{w}-\tilde{w}_{0} \text { weakly in } H^{1,2}(\tilde{\Omega}) \text { and strongly in } C^{\alpha}(\overline{\tilde{\Omega}}) .
$$

Next we fix two arbitrary points on the central axis between the well and the top of the free boundary: i.e.

$$
x_{\kappa}=\left(0,0, z_{\kappa}\right), \quad k=1,2, \quad \text { with } u_{T}<z_{1}<z_{2}<h .
$$




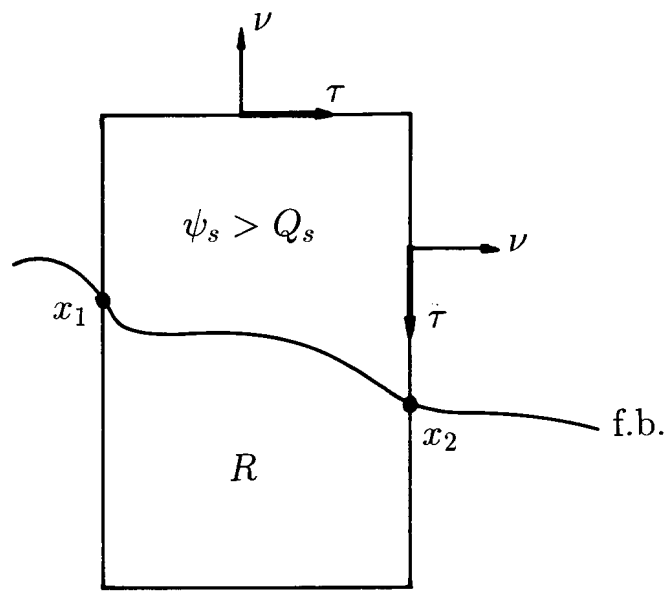

FIG. 25. The rectangle $R$.

Further consider for approriate $Q_{s} \searrow 0$, the sequences of free boundary points

$$
x_{k}^{Q_{s}}=\left\{\psi_{s}=Q_{s}\right\} \cap\left\{x_{3}=z_{k}\right\} \quad \text { for } k=1,2
$$

satisfying

$$
\tilde{w}_{s}\left(x_{1}^{Q_{s}}\right) \geqslant \tilde{w}_{s}\left(x_{2}^{Q_{s}}\right) \text {, }
$$

and with no free boundary points between $x_{\kappa}$ and $x_{\kappa}^{Q_{s}}$. Continuity of the free boundaries ensures the existence of such sequences. Moreover, the monotonicity in $Q_{s}$ of the free boundaries imply that both sequences move monotonically in the direction of the axis. In fact

$$
\lim _{Q_{s} \searrow 0} x_{\kappa}^{Q_{s}}=x_{\kappa}
$$

otherwise the limit free boundary would be above $x_{3}=u_{T}$. The convergence properties of $x_{\kappa}^{Q_{s}}$ and $\tilde{w}_{s}$ imply

$$
\tilde{w}\left(x_{1}\right) \geqslant \tilde{w}\left(x_{2}\right)
$$

Now suppose there exists $x_{0}=\left(0,0, z_{0}\right)$, with $u_{T}<z_{0}<h$, such that $\tilde{w}\left(x_{0}\right)=0$. Then, by (7.10), $\tilde{w}=0$ on the axis between the free boundary and $x_{0}$. Since $\tilde{w}$ is harmonic in a neighbourhood of that segment and $\nabla \tilde{w} \cdot e_{r} \leqslant 0$, we obtain a contradiction.

\section{REFERENCES}

1. Alt, H. W. Strömungen durch inhomogene poröse Medien mit freiem Rand. J. Reine Ang. Math. 21, (1979) 89-115. 
2. Alt, H. W., Caffarelli, L. A., \& Friedman, A. The Dam problem with two fluids. Commun. Pure Appl. Math. 37, (1984) 601-645.

3. Alt, H. W., Caffarelli, L. A., \& Friedman, A. Variational problems with two phases and their free boundaries. Trans. AMS 282, (1984) 431-459.

4. Alt, H. W. \& VAN DUIJn, C. J. A stationary flow of fresh and salt groundwater in a coastal aquifer. Nonlinear Anal. Theory Methods Appl 14, (1990) 625-656.

5. Alt, H. W. \& VAN DUIJn, C. J. A free boundary problem involving a cusp. Part I: global analysis. Eur. J. Appl. Math. 4, (1993) 39-63.

6. Alt, H. W. \& VAN DuiJn, C. J. A free boundary problem involving a cusp. Part II: local analysis. Adv. Math. Sci. Appl. 8, (1998) 845-900.

7. Brezis, H., Kinderlehrer, K., \& Stampacchia, G. Sure une nouvelle formulation du problème lécoulement à travers une digue. C. R. Acad. Sci. Paris 287, (1978) 711-714.

8. Courant, R. Dirichet's Principle, Conformal Mapping and Minimal Surfaces. Springer-Verlag, New York (reprint 1977).

9. Gilbarg, D. \& Trudinger, N. S. Elliptic Differential Equations of Second Order. Grundlehren der mathematischen Wissenschaft 224. Springer-Verlag, Berlin (1977).

10. Polya, G. \& SzegÖ, G. Aufgaben und Lehrsätze aus der Analysis II. Die Grundlehren der mathematischen Wissenschaften in Einzeldarstellungen. Springer-Verlag, Berlin (1964).

11. Trudinger, N. S. On the comparison principle for quasilinear divergence structure equations. Arch. Ration. Mech. Anal. 57, (1974) 128-133.

12. YIH, C. S. A transformation for free surface flow in porous media. Phys. Fluids 7, (1964) 20-24. 